\title{
Transmisión de volatilidad en el Mercado Integrado Latinoamericano (MILA): una evidencia del grado de integración
}

\author{
FueNTES VÉLEZ, MARIANA \\ Universidad Eafit (Colombia) \\ Correo electrónico: mfuente1@eafit.edu.co \\ Pinilla BarRera, Alejandro \\ Universidad Eafit (Colombia) \\ Correo electrónico: apinill2@eafit.edu.co
}

\begin{abstract}
RESUMEN
Este trabajo da cuenta del avance en la integración del Mercado Integrado Latinoamericano (MILA), mediante el estudio de la relación dinámica entre las volatilidades de los mercados que lo componen: Colombia, México, Perú y Chile. Para esto, se usaron datos, desde 2002 hasta 2018, de los índices accionarios representativos de cada una de las bolsas de valores de los países miembros. Debido a las características particulares que tienen las series financieras, como la no estacionariedad y la varianza dinámica a lo largo del tiempo, se aplican las técnicas de series de tiempo, específicamente, los modelos de la familia GARCH con un enfoque multivariado que permitan capturar la relación existente entre los mercados. Se encontró que individualmente las series son procesos integrados de orden 1 y presentan efectos ARCH. Si bien la existencia de interdependencia de volatilidad entre los mercados es latente y varía a lo largo del tiempo, los resultados de este estudio muestran que esta relación no presenta un aumento significativo después de la conformación del MILA. Así, pese a que los mercados están interrelacionados, la interdependencia no es tan fuerte y, por lo tanto, cada uno de los mercados mantiene fuerte independencia del resto. En otras palabras, la integración no se ha alcanzado completamente y, con ello, las ventajas de esta solo se han evidenciado parcialmente en cada una de las bolsas.
\end{abstract}

Palabras clave: MILA, Modelos GARCH Multivariados, integración bursátil, transmisión de volatilidad, Mercados Latinoamericanos.

Clasificación JEL: C32; G15; C58.

MSC2010: 62P05; 62P20; 91G70.

Artículo recibido el 24 de junio de 2019 y aceptado el 2 de julio de 2020 . 


\title{
Transmission of volatility in the Latin American Integrated Market (MILA): evidence of the degree of integration
}

\begin{abstract}
This paper presents the progress in the integration of the Latin American Integrated Market (MILA by its Spanish acronym) by studying the dynamic relationship between the volatilities of the markets that conform it: Colombia, Mexico, Peru and Chile. To achieve this objective, data between 2002 and 2018 from the stock exchanges' representative indices of each MILA member was used. Due to the particular characteristics of the financial series, such as nonstationarity and dynamic variance over time, time series techniques were applied, specifically, the models of the GARCH family with a multivariate approach captures the relationship existing between markets. It was found that individually all series are integrated processes of order 1 and present ARCH effects. Even though the existence of interdependence of volatility between markets is latent and varies over time, the results of this study show that this relationship does not represent a significant increase after the conformation of the MILA. This fact suggests that, although the markets are interrelated, the interdependence is not strong and therefore each of the markets maintain independence. The integration has not been fully achieved and the advantages of this have only been partially shown in each of the stock exchanges.
\end{abstract}

Keywords: MILA, Multivariate GARCH Model, stock exchange integration, volatility transmission, Latin American Stock Markets.

JEL classification: C32; G15; C58.

MSC2010: 62P05; 62P20; 91G70.

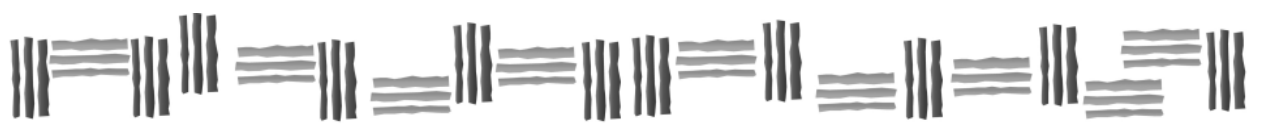




\section{Introducción.}

Las economías de Colombia, Perú, Chile y México se consideran entre las más prometedoras de los países emergentes latinoamericanos. Sin embargo, sus mercados accionarios no están tan desarrollados como los de otros países de la misma categoría, por ejemplo, Brasil. Esta situación también es vivida por países desarrollados como Francia, cuya bolsa de valores no es tan avanzada como la estadounidense. Estas diferencias en el ámbito bursátil hacen necesaria la creación de mecanismos de integración de las bolsas para alcanzar niveles similares a los de países con un mercado bursátil más desarrollado. Algunos de los procesos de integración que han tenido éxito son: EURONEXT, constituido en el 2000 por las bolsas de valores de Ámsterdam (Holanda), París (Francia) y Bruselas (Bélgica), al que luego se le suman Lisboa y Oporto (Portugal); y OMX Nordic, conformado en el año 2004 por las bolsas de valores de Estocolmo (Suecia), Reikiavik (Islandia), Helsinki (Finlandia) y Copenhague (Dinamarca).

Ante este panorama, Colombia, Perú y Chile, en el 2009, inician el primer proceso de integración bursátil en Latinoamérica, dando origen al Mercado Integrado Latinoamericano (MILA). Posteriormente, en el año 2014, se les sumaría México. El objetivo era aumentar la liquidez, la diversificación, la capitalización bursátil, el número de emisores y los volúmenes de transacción que permitieran aprovecharse de la complementariedad de los mercados (Agudelo, Barraza, Castro \& Mongrut, 2012). Es importante destacar que la integración mantuvo la independencia de cada mercado en aspectos normativos y regulatorios, y las negociaciones se realizaron en la moneda respectiva de cada país.

El objetivo de este trabajo es establecer empíricamente si se tiene evidencia de que la integración efectivamente ha sucedido, y si se ha logrado que el MILA se convierta en algo más que la suma de sus partes. Dicho de otro modo, se busca determinar si se ha materializado la idea de un mercado cuya dinámica de crecimiento y desarrollo esté en la trayectoria seguida por los mercados de Brasil o Estados Unidos. Para contrastar la hipótesis del trabajo, este artículo estudiará la transmisión de volatilidad que ocurre en los mercados, ya que, se espera a priori que, si la integración efectivamente ha ocurrido, la transmisión de volatilidad de los mercados debe ser mayor a la que se tenía antes de la conformación del MILA. Por esta razón, se recurre a la metodología del GARCH multivariado que permite: capturar y medir los choques de volatilidad que afectan a cada uno de los mercados de estudio y determinar si los choques de las distintas bolsas de valores están relacionados y en qué magnitud.

El artículo se compone de cinco partes: en primer lugar, se ofrece una revisión de los estudios previos que se han realizado al respecto; luego, se explica la metodología a seguir y la sustentación teórica del modelo empleado; en tercer lugar, se presenta un análisis de los datos que se recolectaron de los rendimientos semanales de las bolsas de valores de los miembros del MILA y se postula el modelo desarrollado en este estudio; posteriormente, se presentan los resultados obtenidos y, finalmente, las conclusiones.

\section{Revisión de la literatura.}

Desde el punto de vista de los mercados, Tamanes (1990) considera que una integración económica es: "un proceso a través del cual dos mercados previamente separados y de dimensiones unitarias estimadas se unen para formar un mercado único (mercado común) de una dimensión adecuada" (p.170). Diversos trabajos acerca de integración de mercados financieros, en su mayoría enfocado a países desarrollados, han permitido evidenciar las ventajas de la integración, así como las transmisiones de volatilidades y los efectos de los choques de volatilidad en estos. Sin embargo, la consolidación del MILA despertó el interés de muchos académicos de estudiar el mercado latinoamericano.

Para los países desarrollados se destacan los estudios realizados por Engle y Susmel (1994), Hamao, Masulis y Ng (1990 y 1991), Fraser y Power (1997), Kanas (1998a) y Fratzscher (2002) quienes 
estudian la integración bursátil internacional examinando lo que se denomina derramamiento de volatilidad entre los mercados. Para esto, los autores utilizan modelos de tipo GARCH tanto univariado como multivariado, en donde, encuentran evidencia empírica a favor de la integración bursátil y la transmisión de volatilidad entre los mercados.

Por otro lado, Kansa (1992), Richards (1995), Choundhry (1996), Baillie y Bollerslev (1989), Kanas (1998b) y Hamori e Imamura (2000) estudian el fenómeno de integración bursátil de los países desarrollados mediante un enfoque de cointegración, encontrando que existen vínculos de interdependencia entre los mercados desarrollados, lo que implica que los mercados bursátiles desarrollados convergen hacia una misma tendencia de largo plazo.

Más recientemente, se destacan los artículos de Piffaut y Miró (2016) y Camargo, Sanabria y López (2012). El primero, mide el efecto contagio entre los mercados de Estado Unidos, Europa y Asia utilizando el modelo de correlaciones dinámicas condicionadas (DCC) de Engle (2002), y concluye que el S\&P 500 está positivamente relacionado con índices europeos y asiáticos (Piffaut y Miró, 2016). El segundo, presenta cómo la incorporación de más países a la Unión Europea aumenta el grado de integración de los mercados financieros, debido a la unificación de la moneda y a la facilidad para invertir en múltiples bolsas. Sin embargo, la crisis griega afecta a los diferentes países de la Unión Europea. Para medir el impacto, utilizan modelos de volatilidad estocástica multivariada de factores aditivos y el filtro de Kalman. Los autores encuentran que los mercados se comportan de manera similar en periodos de alta volatilidad y el rendimiento está explicado en su mayoría por el riesgo sistématico y no por el riesgo ideosincrático. Adicionalmente, los resultados sugieren que los choques internos afectan la estabilidad económica y la integración financiera de la región (Camargo, Sanabria y López, 2012).

Para la presentación de la primera integración financiera de la región latinoamericana, Ariza y Díaz (2015) incluyen un análisis a priori de las economías individuales, con el fin de compararlas con los resultados obtenidos de la integración. Allí, se relata cómo el MILA inicia sus operaciones en el 2011 únicamente con las bolsas de Chile, Colombia y Perú. México se integraría en el año 2014. Entre el 2011 y el 2014, tomando algunos indicadores como el monto negociado de los valores, la capitalización bursátil, los emisores y la cantidad de operaciones, el MILA presenta un incremento considerable de dichos indicadores, alcanzando unos de los objetivos trazados. Con todo, las oportunidades de inversión han sido limitadas debido a que únicamente es posible negociar títulos de renta variable y dado que es necesario ajustar los asuntos tributarios, que representan barreras de entrada e incertidumbre para los inversionistas al igual que el riesgo de tipo de cambio.

Cheng, Firth y Rui (2002) parten de la teoría financiera respecto a las ganancias adicionales obtenidas con la diversificación internacional para estudiar la dependencia de los mercados latinoamericanos. Utilizando la metodología de vectores autorregresivos de Johansen, llegan a la conclusión de que la diversificación es limitada en Latinoamérica porque los mercados están cointegrados y presentan múltiples características comunes que dificultan la variación individual de los mercados. En otras palabras, el hecho de que los mercados bursátiles latinoamericanos se muevan en la misma dirección impide la diversificación del riesgo sistémico local.

Siguiendo esta línea, Cardona, Gutierrez y Agudelo (2015) muestran cómo los países latinoamericanos, cuya integración financiera ha ido aumentando con el tiempo, transmiten las perturbaciones $\mathrm{y}$, en menor proporción, la volatilidad. Esta transmisión va, principalmente, de países grandes a pequeños, por ejemplo, de Estados Unidos a Colombia. No obstante, también hay que mencionar que esta transmisión va de países como Brasil y México a Estados Unidos. Con estas evidencias, se reafirma el rol de liderazgo del mercado brasilero para la región, pero también se destaca la importancia del mercado mexicano. De igual manera, los autores introducen dos formas de transmisión de volatilidad propuestas por Engle, Ito y Lin (1990). De un lado, el planteamiento de "las olas de calor" consiste en que las fuentes de volatilidad son específicas de cada país o región. De otro lado, el planteamiento de "la lluvia de meteoros" alude a la transmisión de volatilidad entre diferentes mercados, países o regiones. No sobra anotar que, respecto a este tema bursátil en Latinoamérica, en el 
artículo se presentan teorías relacionadas con el efecto apalancamiento o asimetría de los retornos (King, Sentana y Wadhwani, 1994).

La formación del MILA representa una diversificación "geográfica y sectorial", por lo que se convierte en una opción atractiva para los inversionistas (Agudelo, Barraza, Castro y Mongrut, 2012, p. 6). En el artículo "Liquidez en los mercados accionarios latinoamericanos: estimando el efecto del mercado integrado Latinoamericano (MILA)", Agudelo, Barraza, Castro y Mongrut (2012) referencian beneficios de la integración encontrados por Schmiedel y Schonenberger (2005) como las externalidades positivas y las economías de escala, que reducen los costos de transacción y se puede apreciar en el aumento de la eficiencia en la negociación de activos. Para demostrar esto, Agudelo, Barraza, Castro y Mongrut (2012) utilizan diferentes medidas de liquidez intradiaria y obtienen una relación directa entre la volatilidad y la liquidez, al igual que los ahorros en los costos de transacción y la actividad bursátil.

Guzmán, Ramírez y Romero (2013), por su parte, analizan la integración financiera entre Chile, Colombia y Perú. Para ello, comparan el portafolio óptimo de cada país con el de la integración y sus principales indicadores, como la rentabilidad. Para esto retoman desde el concepto de integración, incluyendo allí la eliminación de cualquier posibilidad de arbitraje, y los múltiples estudios previos que sostienen las diferentes condiciones para que el mercado sea integrado. Así, los autores referencian trabajos como el de Heston, Rouwenhorst y Wessels (1995), quienes utilizan diferentes indicadores, como la rentabilidad de una acción en diferentes países bajo las mismas condiciones de riesgo o el modelo APT (Arbitrage Pricing Theory). Lo observado por Guzmán, Ramírez y Romero es que sí existe cierto grado de integración y que esta disminuye los beneficios de diversificación, ya que los resultados para el MILA no son significativamente mayores.

Finalmente, el artículo de Lahrech y Sylwester (2011) tiene como objetivo medir la integración de los países de América Latina con Estados Unidos a través de un modelo multivariado de la familia GARCH, específicamente, el modelo de correlación condicional dinámica planteado por Engle (2002). Para la selección del modelo, y como parte de su revisión de literatura, los autores incorporan estudios donde se obtiene como resultado que la correlación no es constante a lo largo del tiempo y sus variaciones son significativas en periodos como la Gran Depresión o finales del siglo XX. Adicional a esto, también utilizan un modelo de transición suavizada para capturar las variaciones estructurales en las correlaciones condicionales. Este resultado es importante porque muestra el cambio del portafolio óptimo explicado por la teoría de la diversificación aplicada en los cambios en las correlaciones. A su vez, estos cambios en las correlaciones pueden ser expuestas a través la transmisión de los choques.

\section{Metodología y estimación.}

Históricamente, la metodología de Box y Jenkins (1970) ha sido la técnica más utilizada para encontrar un modelo que logre recoger el comportamiento tendencial o de largo plazo de una serie financiera, debido a que los índices accionarios son más propensos a tener correlación serial que las acciones individuales (Chan, 1993). Esta metodología modela la serie como un proceso autoregresivo integrado de medias móviles (ARIMA). La especificación de estos modelos, si $y_{t} \sim A R I M A(p, d, q)$ es:

$$
\phi_{p}(L) \Delta^{d} y_{t}=\theta_{q}(L) \varepsilon_{t}
$$

donde $\phi_{p}(L)$ es el polinomio de rezagos de la parte autoregresiva, $\theta_{q}(L)$ es el polinomio de rezagos asociado a la parte de medias móviles y $d$ es el orden de integración de la serie, es decir, el número de veces que se debe diferenciar $y_{t}$ para que sea estacionaria. Diversas pruebas han sido propuestas para encontrar el orden de integración de las series, siendo las más comunes el Dickey-Fuller aumentado (Dickey y Fuller, 1979) y la prueba propuesta por Phillips y Perron (1988). 
- Regresión Dickey-Fuller aumentado:

- Regresión Phillips-Perron:

$$
\Delta y_{t}=\alpha+\beta_{t}+\gamma y_{t-1}+\sum_{i=1}^{p} \delta_{i} y_{t-i}+\mu_{t}
$$

$$
y_{t}=\alpha+\rho y_{t-1}+\mu_{t}
$$

Como lo mencionan Chen, Firth y Meng Rui (2002), los tests difieren en la forma como tratan los problemas de correlación serial. El Phillips-Perron es más robusto a correlaciones seriales y heterocedasticidad. En ambas pruebas la hipótesis nula o a contrastar, es que la serie en cuestión no es estacionaria, es decir, que $\gamma=0$ (en Dickey-Fuller aumentado) o $\rho=1$ (en Phillips-Perron). Por lo tanto, rechazar la hipótesis nula equivale a aceptar que las series son estacionarias.

Una extensión a la metodología de los modelos ARIMA que captura las dinámicas de corto plazo de la varianza del término de perturbación $\varepsilon_{t}$ son los modelos de heterocedasticidad condicional autoregresiva generalizada (GARCH), los cuales, fueron desarrollados inicialmente por Engle (1982) y Bollerslev (1986). De forma general, un proceso $\operatorname{GARCH}(\mathrm{p}, \mathrm{q})$ se representa como:

$$
\begin{gathered}
\varepsilon_{t}=\sqrt{h_{t}} z_{t}, z_{t} \sim N(0,1) \\
h_{t}=\omega+\sum_{i=1}^{p} \alpha_{i} \varepsilon_{t-i}^{2}+\sum_{j=1}^{q} \beta_{j} h_{t-j}
\end{gathered}
$$

La prueba usual para evaluar la presencia de efectos de heterocedasticidad condicional o efectos $\mathrm{ARCH}$, es el test de multiplicadores de Lagrange. El test se divide en dos etapas. En la primera etapa, se regresa la serie en consideración, $r_{t}$, contra una constante $\mu$ :

$$
r_{t}=\mu+\varepsilon_{t}
$$

obteniendo los residuales estimados $\hat{\varepsilon}_{t}$. Posteriormente, se regresa $\hat{\varepsilon}_{t}^{2}$ contra $p$ rezagos de este:

$$
\hat{\varepsilon}_{t}^{2}=\alpha_{0}+\alpha_{1} \hat{\varepsilon}_{t-1}^{2}+\cdots+\alpha_{p} \hat{\varepsilon}_{t-p}^{2}+\mu_{t}
$$

obteniendo el estadístico $T R^{2}$ el cual distribuye $\chi_{p}^{2}$ y donde $T$ es el número de períodos de la serie y $R^{2}$ es el coeficiente de determinación de la última regresión. La hipótesis nula $H_{0}=\alpha_{1}=\cdots=$ $\alpha_{p}=0$, es decir, que $\hat{\varepsilon}_{t}^{2}$ no presenta problemas de correlación y entonces los fenómenos de heterocedasticidad condicional no existen, así, las series son homocedásticas.

Existen especificaciones alternativas de estos modelos que permiten capturar aspectos particulares de las series como la asimetría (Black, 1976), la cual está relacionada con los efectos diferenciados que generan las noticias negativas a las positivas. Algunos de estos modelos son: el SAARCH de Engle (1990); el TGARCH de Zakoian (1994); el GJR-GARCH de Glosten, Jagannathan y Runkle (1993); y el APARCH de Ding, Engle y Granger (1993). En este trabajo se utiliza la especificación del GJR-GARCH para capturar dichos efectos en las series individuales, la cual se muestra a continuación para un GARCH(1,1) GJR-GARCH(1):

$$
h_{t}=\omega+\alpha \varepsilon_{t-1}^{2}+\beta h_{t-1}+\gamma I\left(\varepsilon_{t-1}<0\right) \varepsilon_{t-1}^{2}
$$

donde $\gamma$ captura el efecto asimétrico a través de la variable dummy $\gamma I\left(\varepsilon_{t-1}<0\right)$ que toma el valor de $\$ 1 \$$ cuando la perturbación es negativa y cero en otro caso. 
Los modelos descritos anteriormente resultan efectivos para modelar las series univariadas, sin embargo, cuando se quieren abordar diferentes índices financieros para explicar el comportamiento de uno de ellos en particular y explorar la relación que posee con los otros, el camino adecuado es por medio de modelos híbridos. Por lo tanto, se hace necesario utilizar técnicas que se expliquen de forma multivariada, manteniendo predicciones exactas y conservando la tendencia de los datos sobre el período predicho (Babu y Reddy, 2014).

Siguiendo esta línea de pensamiento y considerando los rasgos idiosincráticos de las series financieras, como la no estacionariedad y la inercialidad, resulta adecuado para este trabajo utilizar los modelos del tipo GARCH multivariado. El modelo GARCH multivariado generaliza el modelo GARCH univariado, permitiendo capturar las interacciones existentes entre distintas series de tiempo a través de las varianzas condicionales y manteniendo las ventajas de su homólogo univariado. En la literatura existen diversas especificaciones para estos modelos que difieren en términos de flexibilidad y parsimonia. Bauwens, Laurent y Rombouts (2006) distinguen tres enfoques diferentes para construir modelos GARCH multivariados:

- Generalizaciones directas del GARCH univariado de Bollerslev (1986), entre las que se destacan: el modelo VECH y el VECH diagonal propuestos por Bollerslev, Engle y Wooldridge (1988); y el modelo BEKK de Baba, Engle, Kraft, y Kroner (1991).

- Combinaciones lineales de modelos GARCH univariados como el modelo GARCH ortogonal propuesto por Alexander y Chibumba (1997) o el modelo GO-GARCH de Van der Weide (2002).

- Combinaciones no lineales de modelos GARCH univariados como el modelo de correlación condicional constante (CCC) de Bollerslev (1990), o el modelo de correlación condicional dinámica (DCC) de Engle (2002).

En este estudio se emplea el enfoque de combinaciones no lineales de modelos GARCH multivariados, particularmente el modelo DCC debido a que presenta dos ventajas claras respecto a los modelos de los otros enfoques. La primera, es que permite escoger una especificación ad hoc del tipo GARCH para cada una de las series consideradas, desde el GARCH estándar hasta el ThresholdGARCH. Esta característica permite gran flexibilidad, ya que podemos escoger el modelo que mejor se ajuste a cada serie, capturando las características particulares de cada una; la segunda ventaja, es que los modelos desde este enfoque son más parsimoniosos, especialmente si se comparan con los modelos VECH y BEKK (Boffelli y Urga 2016). Además, el modelo DCC es preferible respecto a modelos como el CCC porque permite que la correlación condicional varíe a lo largo del tiempo, capturando la dinámica de los mercados en respuesta a las innovaciones y evitando la aparición de sesgo por volatilidad (Celik, 2012). Por estos motivos, autores como Cho y Parhizgari (2008) consideran que este modelo proporciona una medida superior de la correlación.

Por otro lado, desde el punto de vista empírico, la metodología del GARCH multivariado DCC ha sido utilizada en amplios estudios de naturaleza similar, es decir, para estudiar los vínculos entre los mercados financieros y los efectos contagio entre estos. Algunos de los trabajos más importantes son: Celik (2012) estudia el efecto contagio de entre algunos países emergentes; Cho y Parhizgari (2008), y Chiang, Jeon y Li (2007) centran su estudio en Asia; Syllignakis y Kouretas $(2010,2011)$ aplican la metodología para evaluar la integración de los mercados europeos; Najeeb, Bacha, y Masih (2015) investigan los límites en la diversificación de portafolios; Piffaut y Miró (2016) miden el efecto contagio entre Estados Unidos, Europa y Asia; y, Lahrech y Sylwester (2011) miden la integración de los países de América Latina con Estados Unidos.

\subsection{Especificación.}

La especificación de los modelos GARCH multivariados requiere de la correcta especificación de la media y la varianza condicionales. La media condicional se puede especificar de forma general como un modelo VARMA: 


$$
\begin{gathered}
Y_{t}=\Phi_{0}+\Phi_{1} Y_{t-1}+\cdots+\Phi_{p} Y_{t-p}+\varepsilon_{t}+\theta_{1} \varepsilon_{t-1}+\cdots+\theta_{q} \varepsilon_{t-q} \\
\varepsilon_{t}=H_{t}^{1 / 2} z_{t} \\
\operatorname{Var}\left(Y_{t} \mid \Omega_{t-1}\right)=\operatorname{Var}\left(\varepsilon_{t} \mid \Omega_{t-1}\right)=H_{t}^{\frac{1}{2}} \operatorname{Var}\left(z_{t} \mid \Omega_{t-1}\right) H_{t}^{\frac{1}{2}}=H_{t}
\end{gathered}
$$

donde $z_{t}$ es un vector aleatorio independiente e idénticamente distribuido tal que $E\left(z_{t}\right)=0 \mathrm{y}$ $\operatorname{Var}\left(z_{t}\right)=I_{n}$ (matriz identidad de orden $n$ ). $\Omega_{t-1}$ denota la matriz de información en el período $t-1$. Y $H_{t}$ es la matriz de varianzas y covarianzas condicional del proceso. La diferencia entre los distintos modelos GARCH multivariados radica en la especificación de la matriz $H_{t}$. Particularmente, para el modelo DCC usado en este trabajo, la especificación de la matriz $H_{t}$ es:

$$
\begin{gathered}
H_{t}=D_{t} R_{t} D_{t} \\
D_{t}=\operatorname{diag}\left(h_{11, t}^{\frac{1}{2}}, \ldots, h_{n n, t}^{\frac{1}{2}}\right) \\
R_{t}=\operatorname{diag}\left(q_{11, t}^{-\frac{1}{2}}, \ldots, q_{n n, t}^{-\frac{1}{2}}\right) Q_{t} \operatorname{diag}\left(q_{11, t}^{-\frac{1}{2}}, \ldots, q_{n n, t}^{-\frac{1}{2}}\right) \\
Q_{t}=\left(1-\lambda_{1}-\lambda_{2}\right) \bar{Q}+\lambda_{1} \frac{\varepsilon_{t-1}}{\sqrt{h_{t-1}}}\left(\frac{\varepsilon_{t-1}}{\sqrt{h_{t-1}}}\right)^{\prime}+\lambda_{2} Q_{t-1}
\end{gathered}
$$

$D_{t}$ es una matriz diagonal que contiene las desviaciones estándar calculadas por los modelos GARCH univariados de las series. $R_{t}$ es la matriz de correlaciones de $\varepsilon_{t}$ y depende de la matriz $Q_{t}$, la cual, contiene la información acerca de cómo varían las correlaciones a lo largo del tiempo. La idea de Engle es que los elementos de la matriz $Q_{t}$ sigan un proceso GARCH $(1,1)$ univariado. Así, la única restricción que hay que garantizar para que el proceso sea estacionario es que $\lambda_{1}+\lambda_{2}<1$. Con esta especificación, la matriz $\bar{Q}$ resulta ser la matriz de correlaciones incondicional, es decir, la matriz de correlaciones del largo plazo. Una particularidad de este modelo es que en el caso de que $\hat{\lambda}_{1}$ y $\hat{\lambda}_{2}$ resulten ser no significativos, el modelo termina siendo el CCC de Bollerslev.

\subsection{Estimación.}

La estimación de este modelo, generalmente, se realiza en dos etapas. En la primera etapa se estiman los $n$ modelos GARCH univariados de las series para obtener las desviaciones estándar condicionales que componen la matriz $D_{t}$. Después, se hallan los residuales estandarizados como $\zeta_{t}=D_{t}^{-1 / 2} \varepsilon_{t}$ y se procede a hallar la matriz $R_{t}$, donde los parámetros son estimados por máxima verosimilitud (MV). Esta estimación por MV se debe hacer en dos etapas. En la primera, se estiman los parámetros de los modelos univariados y, en la segunda, los parámetros concernientes a la dinámica de las correlaciones. Un estimador ineficiente pero consistente de $R_{t}$ es la matriz identidad. Así, en la primera etapa de la estimación la función de log-verosimilitud es:

$$
\log _{1}\left(\theta_{1}\right)=-\frac{1}{2} \sum_{t=1}^{T}\left[\log \left(\operatorname{det}\left(R_{t}\right)\right)+D_{t}^{-1} \varepsilon_{t}^{2}\right]
$$

donde $\theta_{1}$ es el conjunto de parámetros de todos los modelos GARCH univariados de cada una de las series. La segunda etapa de la estimación requiere maximizar la función de log-verosimilitud:

$$
\log _{1}\left(\theta_{1} \mid \theta_{2}\right)=-\frac{1}{2} \sum_{t=1}^{T}\left[\log \left(\operatorname{det}\left(R_{t}\right)\right)+\left(D_{t}^{-1} \varepsilon_{t}\right)^{\prime R_{t}^{-1}}\left(D_{t}^{-1} \varepsilon_{t}\right)\right]
$$




\subsection{Funciones impulso-respuesta.}

Finalmente, debido a las similitudes de la metodología GARCH multivariada con los procesos VARMA, resulta posible aplicar los análisis de impulso respuesta, introducidos por Sims (1990) para ver el comportamiento de la volatilidad ante un choque. Para ello, han surgido diversos enfoques, por ejemplo, el enfoque de Koop et al. (1996), el de Engel y Ng (1993) o el de Gallan et al. (1993), entre otros. En este trabajo se utilizarán las funciones impulso respuesta generalizadas (GIRF), propuestas por Koop et al. (1996) y Pesaran y Shin (1998). Estas funciones serán aplicadas a la volatilidad de las series, lo que se conoce como VIRF (funciones de impulso-respuesta de volatilidad). La VIRF representa el impacto de un choque en $t$ de la variable $i$ sobre la varianza condicional de la variable $i$ en el período $m$, y se expresa de la siguiente forma:

$$
\operatorname{VIRF}(m, i, j)=E\left(H_{t+m} \mid \varepsilon_{i, t}=\delta_{i, t}, \Omega_{t-1}\right)-E\left(H_{t+m} \mid \varepsilon_{i, t}=0, \Omega_{t-1}\right)
$$

donde $H_{t+m}$ es la matriz de varianzas y covarianzas en el período $t+m ; \delta_{i, t}$ es la magnitud del choque de la variable $i$ en el período $t ; \mathrm{y} \Omega_{t-1}$ representa la información conocida en el período anterior al choque. El problema principal para determinar las VIRFs radica en el cálculo de los pronósticos o valores esperados de las varianzas de las series. Para el modelo DCC, el procedimiento fue detallado por Engle y Sheppard (2001) y consiste en 3 pasos:

1. Usando los modelos univariados de cada una de las series se determina la varianza individual con el fin de construir la matriz $D_{t+m} \mid \Omega_{t}$ descrita en la metodología:

$$
\begin{gathered}
E\left(h_{i, t+1} \mid \Omega_{t}\right)=\omega+\alpha_{i} \delta_{i, t}+\beta_{i} h_{i, t}, m=1 \\
E\left(h_{i, t+1} \mid \Omega_{t}\right)=\omega+\left(\alpha_{i}+\beta_{i}\right) h_{i, t+m-1}, m>1
\end{gathered}
$$

2. Se pronostica la matriz $Q_{t+m} \mid \Omega_{t}$ :

$$
\begin{gathered}
E\left(Q_{t+1} \mid \Omega_{t}\right)=\left(1-\lambda_{1}-\lambda_{2}\right) \bar{Q}+\lambda_{1} \frac{\varepsilon_{t}}{\sqrt{h_{t}}} \frac{\varepsilon_{t}{ }^{\prime}}{\sqrt{h_{t}}}+\lambda_{2} Q_{t}, m=1 \\
E\left(Q_{t+m} \mid \Omega_{t}\right)=\left(1-\lambda_{1}-\lambda_{2}\right) \bar{Q}+\lambda_{1} E\left(\frac{\varepsilon_{t}}{\sqrt{h_{t+m-1}}} \frac{\varepsilon_{t}{ }^{\prime}}{\sqrt{h_{t+m-1}}} \mid \Omega_{t}\right)+\lambda_{2} Q_{t+m-1}, m>1 \\
\text { Donde } E\left(\frac{\varepsilon_{t}}{\sqrt{h_{t+m-1}}} \frac{\varepsilon_{t^{\prime}}}{\sqrt{h_{t+m-1}}} \mid \Omega_{t}\right) \approx E\left(Q_{t+m-1} \mid \Omega_{t}\right)
\end{gathered}
$$

3. Finalmente, se estima la matriz de varianzas y covarianzas condicional:

$$
E\left(H_{t+m} \mid \Omega_{t}\right)=E\left(D_{t+m} \mid \Omega_{t}\right) E\left(R_{t+m} \mid \Omega_{t}\right) E\left(D_{t+m} \mid \Omega_{t}\right)
$$

\section{Hechos estilizados.}

Las series utilizadas son los índices bursátiles de cada una de las bolsas consideradas, los cuales recogen el desempeño del mercado en función de sus activos más representativos (BVC, 2008). Se usan datos semanales del COLCAP (Colombia), IPSA (Chile), IGBVL (Perú) e IPC (México). La información fue obtenida de Bloomberg desde la semana del 19 de julio del 2002 hasta la semana del 18 de mayo del 2018, para un total de 827 datos por cada uno de los índices. Esta frecuencia semanal de los datos captura los efectos de cada uno de los días de la semana, incluyendo el efecto fuerte de los viernes y los lunes, sin suavizarlo tanto como cuando se toman datos con frecuencia mensual. De igual forma, permite homogeneizar los datos para todos los países, porque cada uno de ellos tiene su propio calendario bursátil. En las Figuras 1 y 2 se puede apreciar la evolución de las series en niveles y sus rendimientos a lo largo del tiempo. 
Figura 1. Precios de los índices.

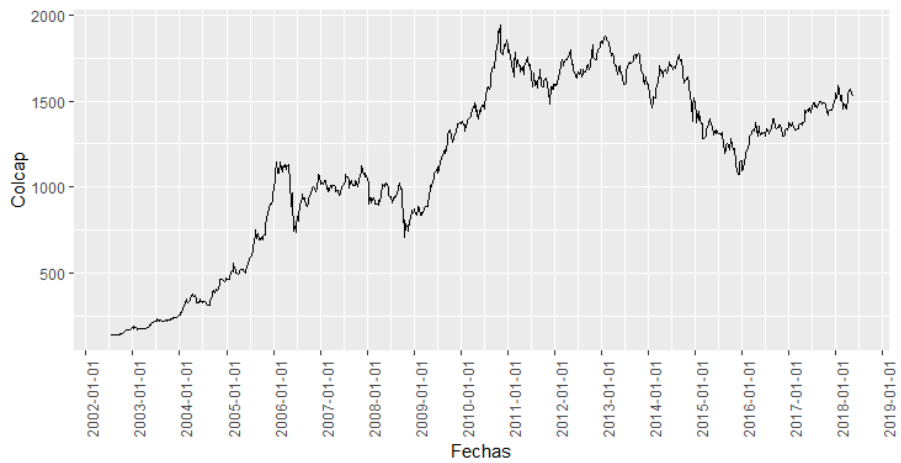

(a) COLCAP

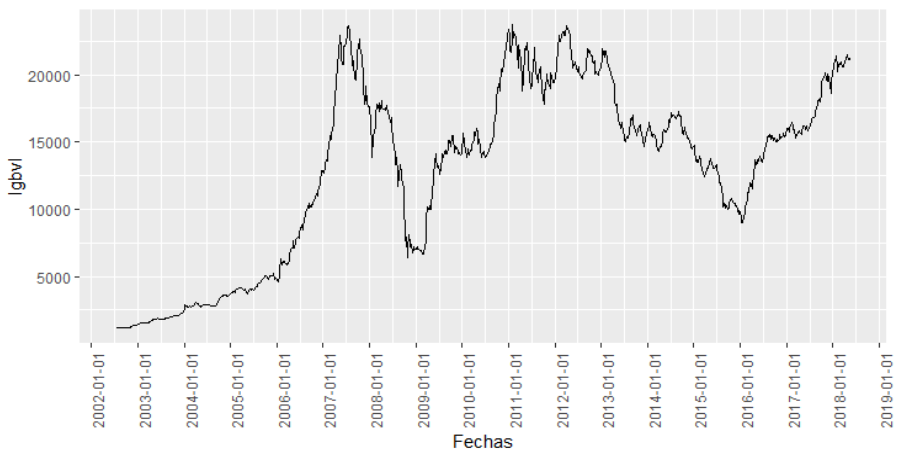

(c) IGBVL

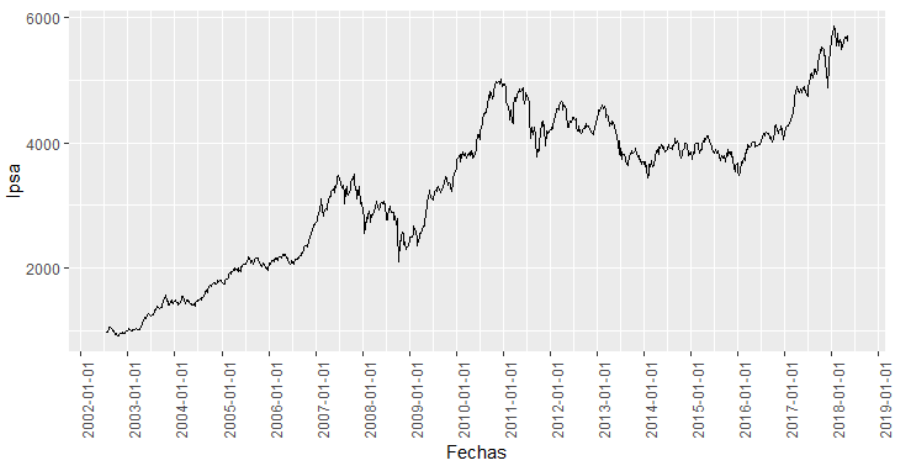

(b) IPSA

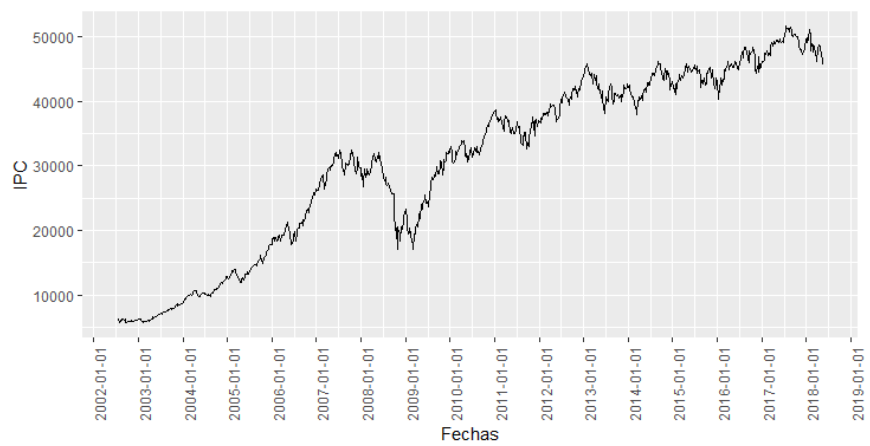

(d) IPC

Fuente: Elaboración propia.

Antes de realizar el análisis econométrico de las series, es importante resaltar y presentar algunos hechos que se pueden observar en las gráficas de las series de los precios de los cuatro países, presentadas en las figuras 1 y 2 . Estos acontecimientos se pueden caracterizar en intervalos de tiempo o como hechos únicos. A modo de ejemplo de los intervalos de tiempo, entre 2002 y 2007, se observa un crecimiento sostenido debido al boom de los commodities en la primera década del nuevo milenio. Sin embargo, el COLCAP tuvo una caída fuerte respecto a los otros índices que conforman el MILA. Dicha caída, ocurrida en junio de 2006, fue una respuesta a la caída generalizada en las principales bolsas del mundo (excluyendo la de Nueva York) por las expectativas negativas de los agentes a las variaciones de las tasas de interés de Estados Unidos y del banco Central Europeo ("Histórica caída", 2006). Cabe destacar que, durante este intervalo de tiempo, el IGBVL presenta un crecimiento en precio superior al COLCAP, IPSA e IPC, alcanzando un máximo histórico en el mes de julio de 2007. Dicho crecimiento en precio fue generado por su entorno macroeconómico favorable: alto crecimiento del PIB, alta inversión pública y privada, e indicadores positivos del sector fiscal. 
Figura 2. Rendimientos de los índices.

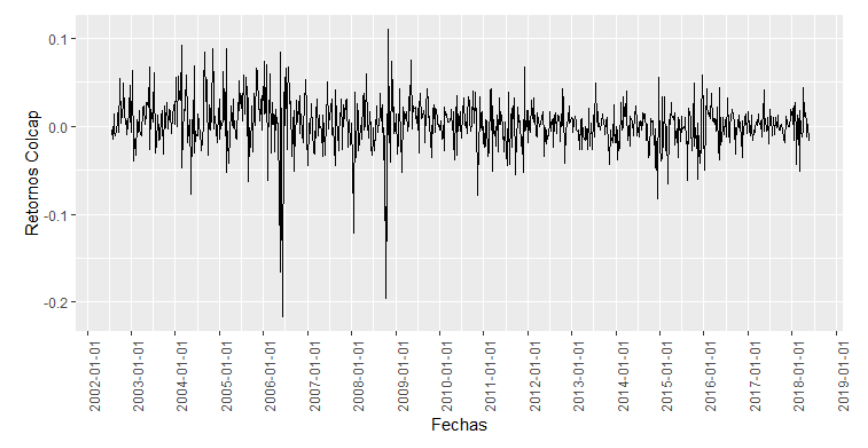

(a) Retornos COLCAP

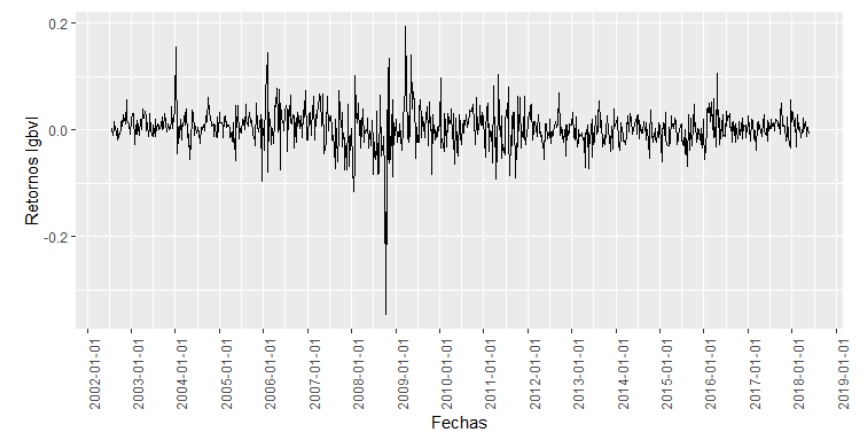

(c) Retornos IGBVL

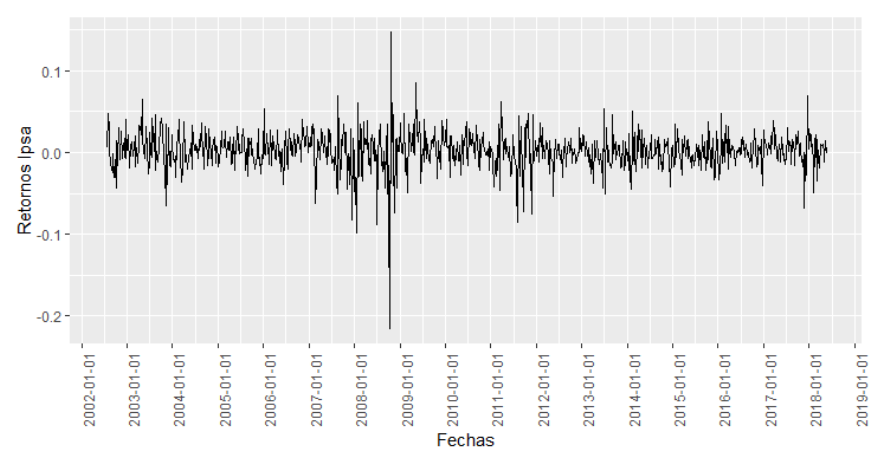

(b) Retornos IPSA

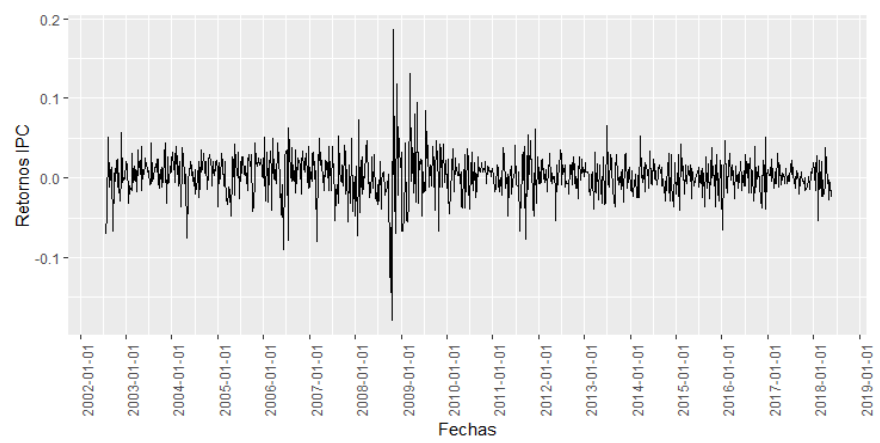

(d) Retornos IPC

Fuente: Elaboración propia.

Para el mes de octubre del 2008, todos los mercados presentan una caída en sus precios y rendimientos negativos por la crisis financiera de Estados Unidos, debido al colapso de la burbuja inmobiliaria. Después de esta histórica caída, el 2009 fue un período de estabilización de los sistemas financieros para todos los mercados, como lo expresa el informe anual de la Bolsa de Valores de Colombia (2009). La intervención tanto del gobierno como de los Bancos Centrales, la creación de políticas, instrumentos y mecanismos logró devolver el nivel del mercado al previo a la crisis y restituir la curva de rendimientos, lo que se observa también en la tendencia creciente de la curva de los precios. Sin embargo, el mercado colombiano y peruano vuelven a sufrir pequeñas caídas progresivas en las cotizaciones a causa de la caída generalizada de los precios de los commodities del año 2014 y alcanzan su punto de inflexión en el año 2016, cuando las cotizaciones del COLCAP y el IGBVL alcanzaron mínimos históricos. Otra noticia que impactó negativamente fue la crisis de la deuda griega, que afectó tanto a las tasas de cambio como la percepción del riesgo de los inversionistas. Estos, en vista de los acontecimientos, prefirieron llevar sus activos a instrumentos más seguros. Este seguimiento de la crisis griega tuvo repercusiones desde la publicación de los primeros reportes hasta las negociaciones.

Finalmente, para el mes de diciembre de 2017, los rendimientos de las bolsas cayeron: los rendimientos del COLCAP, IPSA e IPC, lo hicieron en mayor magnitud que el IGBVL. Ahora, las series presentan recuperaciones hasta mayo del 2018, mostrando una tendencia creciente.

Se puede ver, entonces, cómo los choques externos afectan a los mercados bursátiles latinoamericanos que presentan tendencias similares a lo largo del tiempo. No obstante, algunos de estos afectan con mayor intensidad a unos mercados respecto a otros. Esto es así no solo por la dependencia y relación entre los mercados, sino también por las respuestas de cada uno y el entorno macroeconómico en el que se desarrollan. 
Las estadísticas descriptivas de los retornos en las Tablas 1 y 2 . En estas, se separa la muestra en dos submuestras compuestas por el período antes de la integración y el período después de la misma. En estas estadísticas se puede observar cómo después de la integración el coeficiente de asimetría es más cercano a cero, al igual que la curtosis para todas las series disminuye, acercando un poco las características de las series a la distribución normal, sin comportarse como dicha distribución. De igual forma, es interesante notar que disminuye la desviación estándar de los índices para el segundo período. Posteriormente, se realizan pruebas de normalidad de los datos, entre ellas los tests de Skewness and Kurtosis, Shapiro-Wilk, Shapiro-Francia, Pormanteau, que se resumen en la Tabla 3. En ellos, se constata que los retornos de las bolsas no distribuyen normal al presentar alta curtosis, colas pesadas, leve asimetría y el rechazo de las pruebas de normalidad.

Tabla 1. Principales estadísticas descriptivas de los retornos de los índices antes de la integración, es decir, entre el 2002 y el 2014.

\begin{tabular}{|c|l|c|c|c|}
\hline \multicolumn{5}{|c|}{ Estadísticas Descriptivas } \\
\hline Índice & Media & Desv.Estándar & Coef.Asimetría & Curtosis \\
\hline COLCAP & 0.0040872 & 0.0303598 & -1.378168 & 12.92739 \\
\hline IPSA & 0.0022442 & 0.0255123 & -1.144789 & 14.00843 \\
\hline IPC & 0.0031977 & 0.0297597 & -0.302462 & 9.308290 \\
\hline IGBVL & 0.0043295 & 0.0388523 & -1.162468 & 16.817740 \\
\hline \multicolumn{5}{|c}{ Fuente: Elaboración propia. } \\
\hline
\end{tabular}

Fuente: Elaboración propia.

Tabla 2. Principales estadísticas descriptivas de los retornos de los índices desde la integración, es decir, desde el 2014 hasta el 2018.

\begin{tabular}{|c|c|c|c|c|}
\hline \multicolumn{5}{|c|}{ Estadísticas Descriptivas } \\
\hline Índice & Media & Desv.Estándar & Coef.Asimetría & Curtosis \\
\hline COLCAP & -0.00022 & 0.0206983 & -0.519141 & 4.64924 \\
\hline IPSA & 0.0018908 & 0.0182091 & -0.033926 & 4.43037 \\
\hline IPC & 0.0003553 & 0.0184201 & -0.095903 & 3.545898 \\
\hline IGBVL & 0.001242 & 0.0227554 & 0.213008 & 4.797070 \\
\hline
\end{tabular}

Fuente: Elaboración propia.

Tabla 3. P-valor de los Tests de normalidad aplicado a los rendimientos logarítmicos de cada una de las series.

\begin{tabular}{|c|c|c|c|c|}
\hline Índice & Skewness and kurtosis & Shapiro-Wilk & Shapiro-Francia & Portmanteau \\
\hline rcolcap & 0.0000 & 0.0000 & 0.00001 & 0.0029 \\
\hline ripsa & 0.0000 & 0.0000 & 0.00001 & 0.1402 \\
\hline ripc & 0.0000 & 0.0000 & 0.00001 & 0.0641 \\
\hline rigbvl & 0.0000 & 0.0000 & 0.00001 & 0.0000 \\
\hline
\end{tabular}

Fuente: Elaboración propia.

\section{Resultados.}

Esta sección se divide en tres partes: en la primera parte, se mostrarán los resultados del análisis preliminar de los modelos univariados siguiendo la estructura propuesta en la metodología; luego, se presentarán los resultados de la estimación multivariada, y, finalmente se expondrán las funciones impulso-respuesta. 


\subsection{Análisis preliminar univariado.}

Como se mencionó en la metodología, antes de estimar el modelo multivariado es necesario encontrar el mejor modelo univariado que describa cada serie utilizando la metodología ARIMA-GARCH. El primer paso, es analizar el orden de integración de las series individuales. En la tabla 4 se muestran los resultados del Dickey-Fuller y el Phillips-Perron para las series en niveles de forma logarítmica. Los resultados muestran que no se rechaza la hipótesis nula de no estacionariedad para todos los índices accionarios.

Tabla 4. P-valor de los tests de raíces unitarias para las series en logaritmos.

\begin{tabular}{|c|c|c|}
\hline Variables & Dickey-Fuller Aumentado (con 2 rezagos y tendencia) & Phillips-Perron \\
\hline COLCAP & 0.8343 & 0.3463 \\
\hline IPSA & 0.6156 & 0.8032 \\
\hline IPC & 0.4217 & 0.5716 \\
\hline IGBVL & 0.7100 & 0.5054 \\
\hline
\end{tabular}

Fuente: Elaboración propia.

De igual forma, en la Tabla 5 se reportan los resultados de las pruebas, pero considerando los rendimientos logarítmicos. En este caso, la hipótesis de no estacionariedad es rechazada, lo que implica que las series en diferencias son estacionarias y, por tanto, los índices accionarios son integrados de orden $1(I(1))$ como se esperaba a priori.

Tabla 5. P-valor de los tests de raíces unitarias para los rendimientos logarítmicos.

\begin{tabular}{|c|c|c|}
\hline Variables & Dickey-Fuller Aumentado (con 2 rezagos y tendencia) & Phillips-Perron \\
\hline rcolcap & 0,0000 & 0,0000 \\
\hline ripsa & 0,0000 & 0,0000 \\
\hline ripc & 0,0000 & 0,0000 \\
\hline rigbvl & 0,0000 & 0,0000 \\
\hline
\end{tabular}

Fuente: Elaboración propia.

En la Tabla 6 se detallan los resultados de la prueba para establecer efectos ARCH:

Tabla 6. P-valor de la prueba a distintos rezagos y con todos los índices.

\begin{tabular}{|c|c|c|c|c|}
\hline \multicolumn{5}{|c|}{ Test de efectos $\boldsymbol{A R C H}$-LM } \\
\hline Rezagos & COLCAP & IPSA & IPC & IGBVL \\
\hline 1 & 0,0185 & 0,0000 & 0,0000 & 0,0000 \\
\hline 2 & 0,0002 & 0,0000 & 0,0000 & 0,0000 \\
\hline 3 & 0,0000 & 0,0000 & 0,0000 & 0,0000 \\
\hline 4 & 0,0000 & 0,0000 & 0,0000 & 0,0000 \\
\hline 5 & 0,0000 & 0,0000 & 0,0000 & 0,0000 \\
\hline
\end{tabular}

Fuente: Elaboración propia. 
En todas las series y todos los rezagos se rechaza la hipótesis nula y, por tanto, se presentan fenómenos de heterocedasticidad condicional.

Finalmente, se estima el modelo ARIMA-GARCH para cada uno de los índices accionarios. El método adecuado para determinar la especificación correcta de los modelos GARCH es a través de los criterios de información y garantizando la estacionariedad y la no correlación serial de los residuales. A continuación, se presentan los modelos ARMA-GARCH definitivos que se usarán en el modelo multivariado:

$$
\begin{aligned}
& \operatorname{rcolcap}_{t}=\mu_{\text {colcap }}+\sum_{i=1}^{2} \phi_{i, \text { colcap }} \text { rcolcap }_{t-i}+\varepsilon_{t, \text { colcap }} \\
& h_{t, \text { colcap }}=\omega_{\text {colcap }}+\alpha_{\text {colcap }} \varepsilon_{t-1, \text { colcap }}^{2}+\beta_{\text {colcap }} h_{t-1, \text { colcap }} \\
& +\gamma_{\text {colcap }} I\left(\varepsilon_{t-1, \text { colcap }}<0\right) \varepsilon_{t-1, \text { colcap }}^{2} \\
& \operatorname{rigbvl}_{t}=\mu_{i g b v l}+\sum_{i=1}^{4} \phi_{i, i g b v l} \text { rigbvl }_{t-i}+\sum_{j=1}^{4} \theta_{i, i g b v l} \varepsilon_{t-j, i g b v l}+\varepsilon_{t, i g b v l} \\
& h_{t, i g b v l}=\omega_{i g b v l}+\alpha_{i g b v l} \varepsilon_{t-1, i g b v l}^{2}+\beta_{i g b v l} h_{t-1, i g b v l} \\
& \operatorname{ripc}_{t}=\mu_{i p c}+\phi_{1, i p c} \text { ripc }_{t-1}+\varepsilon_{t, i p c} \\
& h_{t, i p c}=\omega_{i p c}+\alpha_{i p c} \varepsilon_{t-1, i p c}^{2}+\beta_{i p c} h_{t-1, i p c}+\gamma_{i p c} I\left(\varepsilon_{t-1, i p c}<0\right) \varepsilon_{t-1, i p c}^{2} \\
& \text { ripsa }_{t}=\mu_{i p s a}+\varepsilon_{t, i p s a} \\
& h_{t, i p s a}=\omega_{i p s a}+\alpha_{i p s a} \varepsilon_{t-1, i p s a}^{2}+\beta_{i p s a} h_{t-1, i p s a}+\gamma_{i p s a} I\left(\varepsilon_{t-1, i p s a}<0\right) \varepsilon_{t-1, i p s a}^{2}
\end{aligned}
$$

Los detalles de estas estimaciones se pueden encontrar en el Anexo.

\subsection{Modelo GARCH multivariado DCC.}

Después del análisis preliminar y siguiendo la metodología descrita anteriormente, se estima el modelo DCC multivariado. Los resultados de las estimaciones se presentan en la tabla 7 La mayoría de los coeficientes resultan estadísticamente significativos, sobre todo, los asociados a la especificación de la correlación dinámica multivariada. Esto valida lo esperado a priori: que la correlación es dinámica y no constante a lo largo del tiempo. Los valores de los coeficientes $\lambda_{1}$ y $\lambda_{2}$ son de 0.02 y 0.97 , respectivamente, lo cual indica que la matriz de correlaciones es altamente persistente pues depende mucho de la matriz rezagada $Q_{t-1} \mathrm{y}$, en menor medida, de los choques pasados.

Tabla 7. Resultados del modelo DCC estimado, en el cual se muestran los coeficientes, los errores estándar y el p-valor.

\begin{tabular}{|c|c|c|c|c|}
\hline \multicolumn{5}{|c|}{ Modelo DCC } \\
\hline Índice & Variable & Coeficiente & Desv. Estándar & P-valor \\
\hline \multirow{7}{*}{ rcolcap } & $\mu$ & 0.002830856 & 0.000935 & 0.002467 \\
\cline { 2 - 5 } & $\phi_{1}$ & 0.07747752 & 0.047314 & 0.101520 \\
\cline { 2 - 5 } & $\phi_{2}$ & 0.1066815 & 0.048147 & 0.026710 \\
\cline { 2 - 5 } & $\omega$ & 0.000100 & 0.000049 & 0.042555 \\
\cline { 2 - 5 } & $\alpha$ & 0.1939657 & 0.059774 & 0.001174 \\
\cline { 2 - 5 } & $\beta$ & 0.6436487 & 0.100123 & 0.000000 \\
\hline rigbvl & $\gamma$ & 0.1241257 & 0.160899 & 0.440439 \\
\hline
\end{tabular}




\begin{tabular}{|c|c|c|c|c|}
\hline & $\phi_{1}$ & 0.4030216 & 0.035417 & 0.000000 \\
\hline & $\phi_{2}$ & 0.1679684 & 0.010883 & 0.000000 \\
\hline & $\phi_{3}$ & 0.9316687 & 0.010900 & 0.000000 \\
\hline & $\phi_{4}$ & -0.5218204 & 0.036244 & 0.000000 \\
\hline & $\theta_{1}$ & -0.2691925 & 0.001933 & 0.000000 \\
\hline & $\theta_{2}$ & -0.1140603 & 0.002313 & 0.000000 \\
\hline & $\theta_{3}$ & -0.9449963 & 0.000496 & 0.000000 \\
\hline & $\theta_{4}$ & 0.3828108 & 0.001693 & 0.000000 \\
\hline & $\omega$ & 0.000090 & 0.000057 & 0.117060 \\
\hline & $\alpha$ & 0.2986567 & 0.149626 & 0.045932 \\
\hline & $\beta$ & 0.6553163 & 0.151327 & 0.000015 \\
\hline \multirow{6}{*}{ ripc } & $\mu$ & 0.002032525 & 0.000764 & 0.007841 \\
\hline & $\phi_{1}$ & -0.06722044 & 0.038441 & 0.080347 \\
\hline & $\omega$ & 0.000032 & 0.000018 & 0.083549 \\
\hline & $\alpha$ & 0.04042694 & 0.028674 & 0.158579 \\
\hline & $\beta$ & 0.8480823 & 0.053995 & 0.000000 \\
\hline & $\gamma$ & 0.1150129 & 0.063064 & 0.068190 \\
\hline \multirow{5}{*}{ ripsa } & $\mu$ & 0.002122061 & 0.000703 & 0.002537 \\
\hline & $\omega$ & 0.000051 & 0.000054 & 0.346845 \\
\hline & $\alpha$ & 0.05092518 & 0.037880 & 0.178823 \\
\hline & $\beta$ & 0.7532088 & 0.183715 & 0.000041 \\
\hline & $\gamma$ & 0.1912115 & 0.149434 & 0.200695 \\
\hline \multirow{2}{*}{ DCC } & $\lambda_{1}$ & 0.01609859 & 0.012512 & 0.198227 \\
\hline & $\lambda_{2}$ & 0.9658611 & 0.041483 & 0.000000 \\
\hline
\end{tabular}

Fuente: Elaboración propia.

La Figura 3 muestra las varianzas condicionales estimadas de cada uno de los índices. Hay que resaltar que todos presentan volatilidades altas en el período de la crisis del 2009. Cabe destacar, el pico del mercado colombiano en el año 2006, que corresponde a la mayor caída de la bolsa colombiana debido a la incertidumbre respecto a las tasas de interés de la FED, que buscaba en esa época controlar brotes inflacionarios. De igual forma, la Unión Europea acababa de subir los tipos de interés, lo que llevó a los mercados de incertidumbre a entrar en nerviosismo. El mercado peruano y mexicano presentan comportamientos parecidos con pequeños picos a lo largo del nuevo milenio y un pico significativo por la crisis de los mercados financieros a nivel mundial. Finalmente, se destaca la estabilidad del mercado chileno sobre el resto de los miembros del MILA.

En la Figura 4, se muestran las correlaciones condicionales dinámicas obtenidas por el modelo. De forma general, es notorio que oscilan entre 0.1 y 0.65 aproximadamente, con una correlación media entre 0.3 y 0.4. Como argumentan Lahrech y Sylwester (2016), Blyn (1973), y Heytens (1986) correlaciones en estos rangos se pueden asociar a una integración débil de los mercados, ya que, se espera a priori que dichas correlaciones sean superiores, por lo menos, a 0.6. 
Figura 3. Varianza condicional de cada uno de los índices.

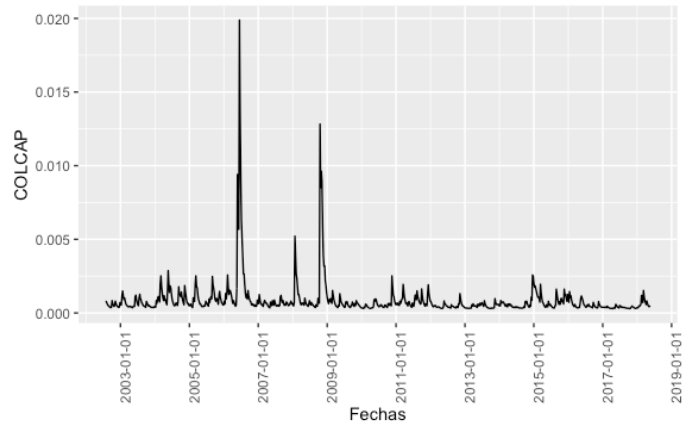

(a) COLCAP

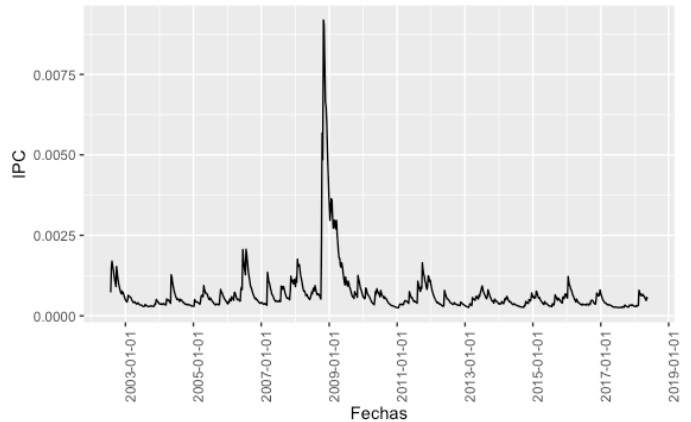

(c) IPSA

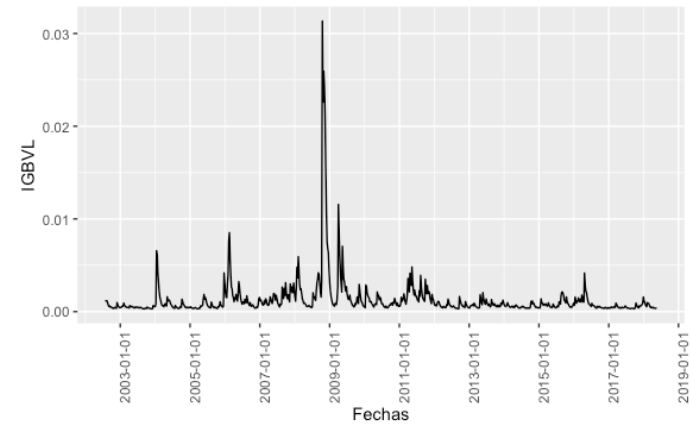

(b) IGBVL

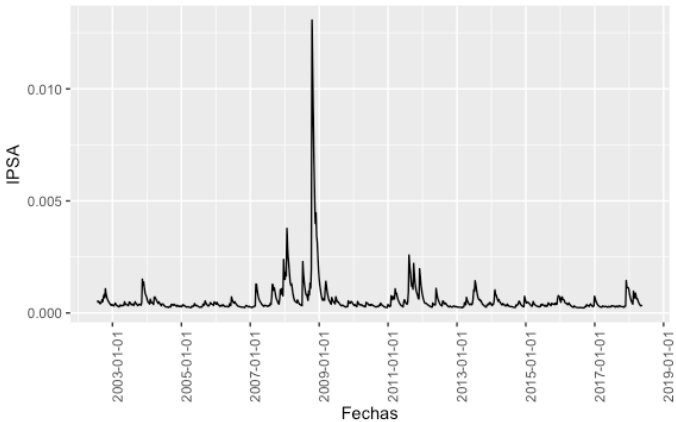

(d) IPC

Fuente: Elaboración propia.

Figura 4. Correlación condicional dinámica de todos los índices.

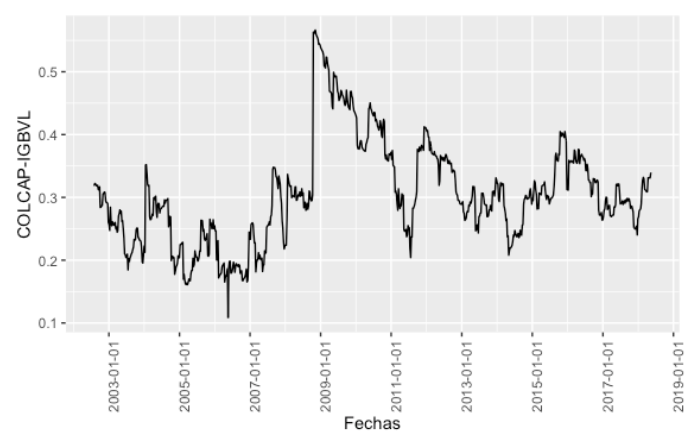

(a) COLCAP-IGBVL

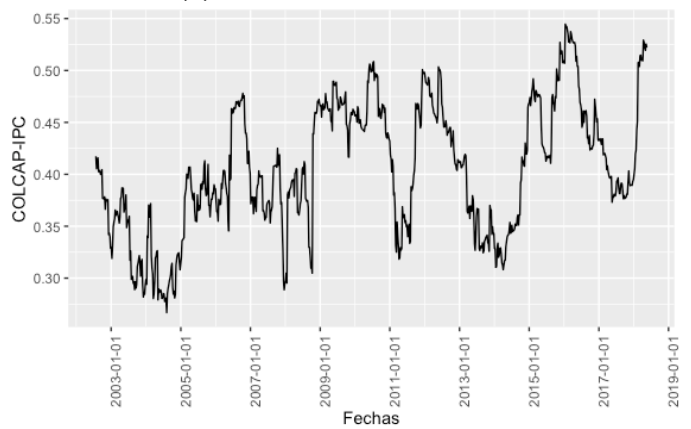

(c) COLCAP-IPC

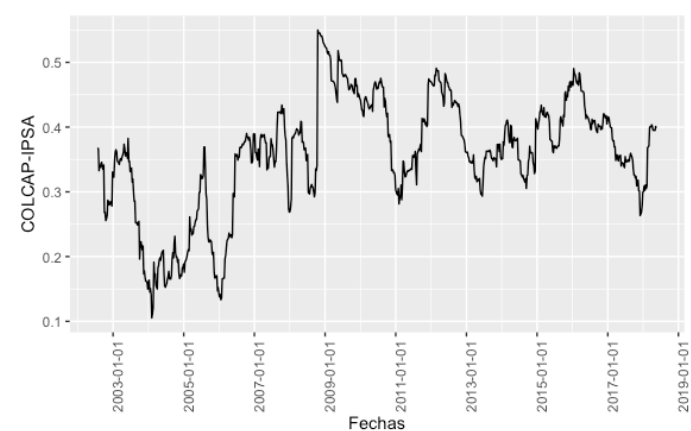

(b) COLCAP-IPSA

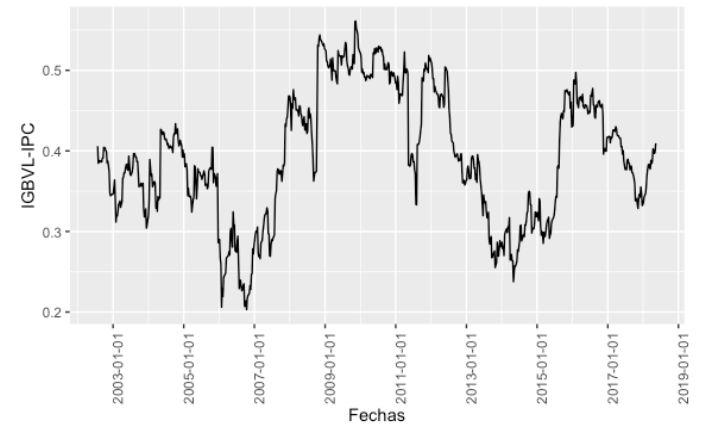

(d) IGBVL-IPC 


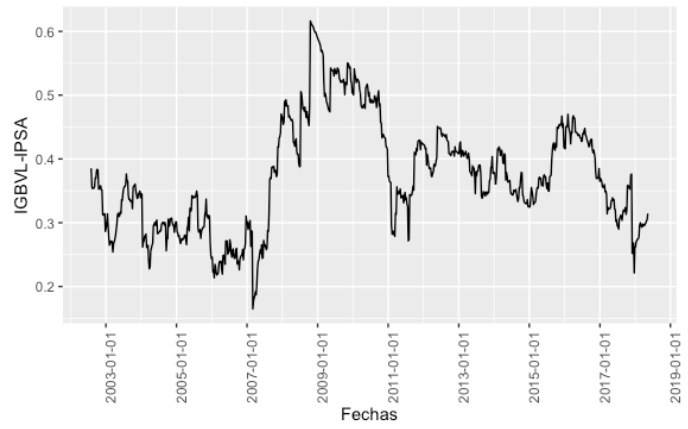

(e) IGBVL-IPSA

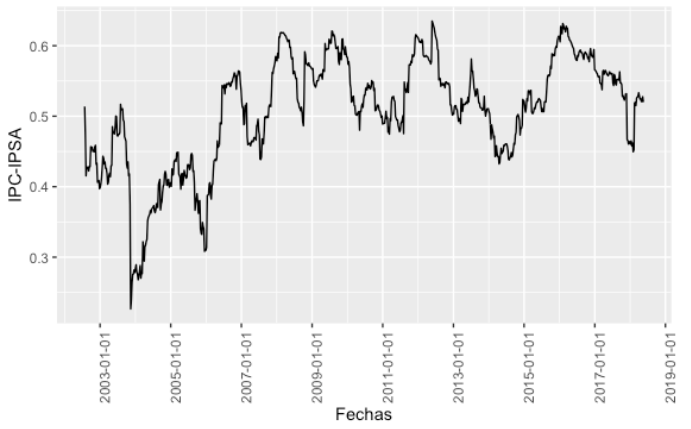

(f) IPSA-IPC

Fuente: Elaboración propia.

Si bien el mercado colombiano está altamente relacionado con el mercado mexicano, la correlación entre ambos países es muy volátil a lo largo del tiempo. Respecto al mercado chileno y peruano, el mercado colombiano presenta más estabilidad en la correlación. Perú muestra el vínculo más débil con el mercado colombiano. Acerca del mercado peruano, es destacable la alta relación que mantiene con Chile, llegando a niveles superiores a 0.6. El mercado chileno se correlaciona bien con todos, pero la relación con el mercado mexicano es la más alta (alrededor de 0.65 en su punto máximo). Finalmente, el mercado mexicano muestra buena correlación y estabilidad con el mercado chileno, a diferencia de la correlación con el colombiano y el peruano.

Por otro lado, la evolución de las correlaciones refleja los comovimientos de los países durante los eventos claves mencionados en el análisis preliminar. De 2002 a 2007, cuando tuvo lugar el boom de los commodities, las correlaciones aumentaron hasta alcanzar puntos máximos entre 0.55 y 0.6 . Esto se debe a que las economías de los cuatro mercados comparten características como la alta dependencia a la exportación de materias primas. Posteriormente, durante las crisis del 2008-2009, las correlaciones alcanzan sus máximos históricos para después caer a los niveles promedio. Luego, con la crisis de los commodities de los años 2014-2016 las correlaciones caen y recuperan sus niveles normales en 20172018. Hay que destacar que la estabilidad en el coeficiente de correlación del mercado mexicano y chileno hace que sean más robustos a los choques de volatilidad que el resto. Estos resultados implicarían que, en épocas de crisis, donde se esperaría a priori que sean de alta volatilidad, los mercados tienen comportamientos divergentes: por un lado, puede aumentar la correlación entre estos, como sucedió en el 2009; o pierden su relación y la transmisión se da en menor medida, como ocurrió con la caída generalizada de los precios de los commodities en el 2014. Por otro lado, cuando las economías de los países están en auge y se espera menor volatilidad, las correlaciones aumentan, haciendo que los efectos de la transmisión se vuelvan significativos.

\subsection{Funciones de impulso-respuesta.}

En la Figura 5 se muestran los resultados de las funciones de impulso respuesta ante un choque negativo de una desviación estándar en cada serie. A priori se espera que el choque de volatilidad aumente las varianzas de todos los mercados debido a que los resultados del modelo muestran evidencia empírica a favor de una relación positiva entre las varianzas de estos. Según la gráfica, el índice más sensible al choque es el IPSA, el cual aumenta su varianza o riesgo en un $20 \%$, aproximadamente. Por otro lado, el más robusto resulta ser el IGBVL, pues su volatilidad solo aumenta $12 \%$, aproximadamente. Este hecho puede ser causado por la carencia de fenómenos de asimetría en la varianza. No obstante, el efecto del choque en el mercado peruano es más duradero que en el mercado colombiano. El IPC y el COLCAP muestran cambios similares en magnitud, dado que ambos aumentan su volatilidad en $15 \%$ aproximadamente. El mercado chileno elimina el efecto del choque más rápidamente en comparación con los otros mercados. Adicionalmente, como es posible observar en las gráficas, los efectos de los choques de volatilidad perduran al menos 15 semanas después de su ocurrencia y, en algunos casos, más de 20 semanas. 


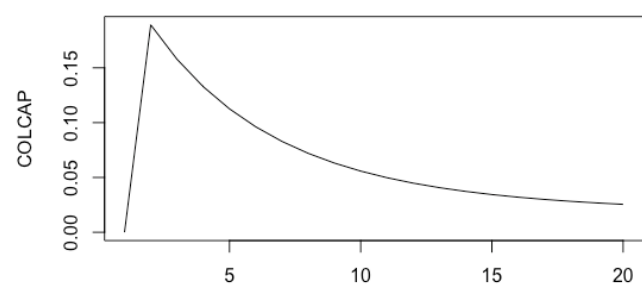

(a) COLCAP

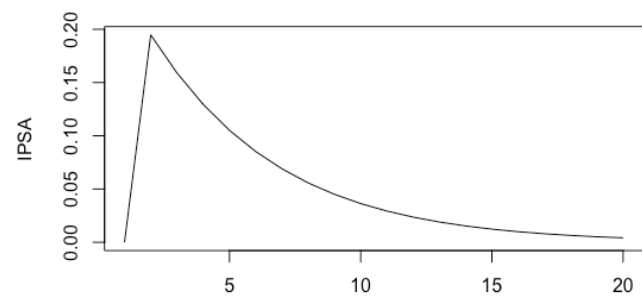

(c) IPSA

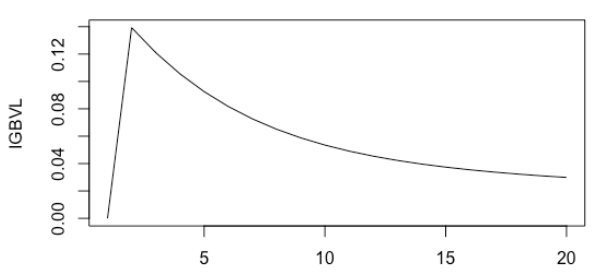

(b) IGBVL

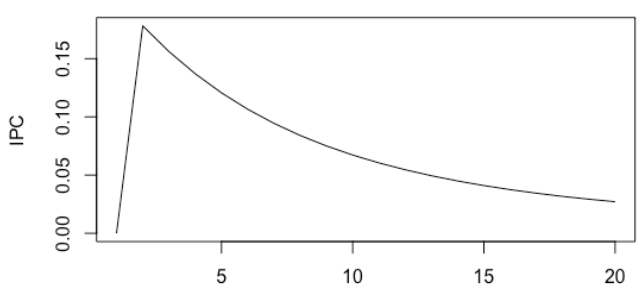

(d) IPC

Fuente: Elaboración propia.

\section{Conclusiones.}

En este estudio, se estimó el modelo GARCH multivariado DCC de Engle (2002), con el fin de encontrar existencia de fenómenos de transmisión de volatilidad que sirvieran como evidencia para el estudio de la integración de los mercados que componen el MILA. Los resultados indican que, efectivamente, existe transmisión de volatilidad entre todos los mercados de forma positiva. Así se observó en las correlaciones estimadas entre las varianzas y las funciones de impulso respuesta. Se encontró que el mercado peruano es más robusto ante choques de volatilidad por la carencia de efectos asimétricos en la varianza. Adicionalmente, se encontró que el mercado chileno es más sensible que los otros, pero los choques en este mercado perduran menos en el tiempo. De igual forma, se observó que, en los tiempos de crisis, las correlaciones seriales entre las volatilidades de los índices aumentaron significativamente, mientras que, en tiempos de estabilidad, disminuyeron. Este resultado implica que las oportunidades de diversificación de portafolios en épocas de crisis son limitadas. De esta manera, se confirma lo planteado por Rui, Cheng y Firth (2002) y pone en entredicho lo presentado por Agudelo et al. (2012).

Cabe mencionar el bajo nivel de las correlaciones, entre 0.1 y 0.6. Se esperaba que fueran más altas por la constitución del MILA. De ahí que las ventajas de la integración no se han evidenciado completamente. Esto se explica por la independencia en términos normativos, regulatorios y monetarios.

Este estudio se diferencia respecto a otros trabajos encontrados en la literatura en varios aspectos. Primero, el enfoque es hacia mercados subdesarrollados, particularmente, los latinoamericanos. Segundo, este estudio se concentra en la dinámica de las volatilidades de los mercados, a diferencia de otros que estudian otras características de estos. Tercero, la metodología empleada es más pertinente en 
términos de la especificación del modelo, porque no implica hacer supuestos explícitos acerca de cómo funciona la transmisión de volatilidad y, respecto a la mayoría de los estudios previos, no se asume una misma especificación para cada una de las series consideradas. Finalmente, se incorporan las funciones impulso respuesta en el modelo DCC de Engle (2002).

Para concluir, con relación a la integración del MILA, permanecen aspectos sin resolver, entre otros: el grado de competitividad del MILA respecto a otros mercados desarrollados; el impacto que ha tenido la incorporación de títulos de renta fija y derivados en la negociación; y la medición de las ganancias de la integración en cada uno de los países miembros del MILA. Esto hace del MILA un campo atractivo, aún por explorar, para las finanzas.

\section{Referencias}

Agudelo, D.A., Barraza, S.E., Castro, M.I., \& Mongrut S. (2012). Liquidez en los mercados accionarios latinoamericanos: estimando el efecto del Mercado Integrado Latinoamericano (MILA). Documentos de Trabajos Economía y Finanzas, 12-21. Medellín: Universidad EAFIT, Escuela de Economía y Finanzas, Centro de Investigaciones Económicas y Financieras (CIEF).

Agudelo, D.A., Cardona, L., \& Gutiérrez, M. (2015). Transmisión de Volatilidad en Mercados Accionarios Latinoamericanos [Trabajo de grado de la Maestría en ciencias en Finanzas]. Universidad EAFIT, Medellín.

Ariza, D.M., \& Díaz, M.F. (2015). Mercado Integrado Latinoamericano (MILA): ¿Objetivos No Cumplidos? [Trabajo de Grado de la Maestría en Administración]. Universidad EAFT, Bogotá.

Baba, Y., Engle, R. F., Kraft, D., \& Kroner, K. F. (1991). Multivariate simultaneous GARCH. Unpublished manuscript, Department of Economics, University of California at San Diego.

Babu, C.N., \& Reddy, B.E. (2014). Selected Indian stock predictions using a hybrid ARIMA-GARCH model. 2014 International Conference on Advances in Electronics Computers and Communications, $1-6$.

Baillie, R.T., \& Bollerslev, T. (1989). The message in daily exchange rates: A conditional variance tale. Journal of Business and Economic Statistics, 7, 297-305.

Bauwens, L., Laurent, S., \& Rombouts, J.V.K. (2006). Multivariate GARCH models: A survey. Journal of Applied Econometrics, 21, 79-109.

Black, F. (1976). The pricing of commodity contracts. Journal of Financial Economics, 3, 167-179.

Blyn, G. (1973). Price series correlation as a measure of market integration. Indian Journal of Agricultural Economics, 28 (902-2018-2204), 56-59.

Boffelli, S., \& Urga, G. (2016). Financial econometrics using Stata. Stata Press Publication.

Bollerslev, T. (1986). Generalized autoregressive conditional heteroskedasticity. Journal of Econometrics, 31, 307-327.

Bollerslev, T. (1990). Modelling the coherence in short-run nominal exchange rates. A multivariate generalized ARCH model. Review of Economics and Statistics, 72, 498-505.

Bollerslev, T., Engle, R.F., \& Wooldridge, J.M. (1988). A capital asset pricing model with time-varying covariances. Journal of Political Economy, 96, 116-131. 
Bolsa de Valores de Colombia (2008). Índices bursátiles [en línea]. Recuperado de https://www.bvc.com.co/pps/tibco/portalbvc/Home/Mercados/

descripciongeneral/indicesbursatiles?action=dummy.

Bolsa de Valores de Colombia (2009). Reporte Anual:2009. Bogotá (Colombia): Bolsa de Valores de Colombia.

Box, G.E.P., \& Jenkins, G.M. (1970). Time Series Analysis: Forecasting and Control. San Francisco, EEUU: Holden-Day.

Box, G.E.P., \& Ljung, G.M. (1978). On a Measure of Lack of Fit in Time Series Model. Biometrika, 65(2), 297-303.

Camargo, A.I., Sanabria, T.P., \& López, F. (2012). Integración de los mercados financieros de Europa: El impacto de la crisis soberana de Grecia. EconoQuantum, 10 (2), 8-34.

Celik, S. (2012). The more contagion effect on emerging markets: The evidence of DCC-GARCH model. Economic Modelling, 29(5), 1946-1959.

Chan, K. (1993). Imperfect information and cross-autocorrelation among stock prices. Journal of Finance, 48(4), 1211-1230.

Chen, G., Firth, M., \& Rui, O.M. (2002). Stock market linkages: Evidence from Latin America. Journal of Banking and Finance, 26, 1113-1141.

Chiang, T.C., Jeon, B.N., \& Li, H. (2007). Dynamic correlation analysis of financial contagion: Evidence from Asian markets. Journal of International Money and finance, 26(7), 1206-1228.

Cho, J.H., \& Parhizgari, A.M. (2008). East Asian financial contagion under DCC-GARCH. International Journal of Banking and Finance, 6(1), 17-30.

Choudhry, T. (1996). Interdependence of stock markets: Evidence from Europe from 1920s and 1930s. Applied Financial Economics, 6(3), 243-249.

Ding, Z., Engel, R.F., \& Granger, W. J. (1993). A long memory property of stock market returns and a new model. Journal of Empirical Finance, 1, 83-106.

Dickey, D.A., \& Fuller, W.A. (1979). Distribution of the estimators for autoregressive time series with a unit root. Journal of the American Statistical Association, 74, 427-431.

Engle, R.F. (1990). Discussion: Stock volatility and the crash of '87. Review of Financial Studies, 3, 103-106.

Engle, R.F. (1982). Autoregressive Conditional Heteroscedasticity with Estimates of the Variance of United Kingdom Inflation. Econometrica, 50(4), 987-1007.

Engle, R.F., \& Sheppard, K. (2001). Theoretical and Empirical properties of Dynamic Conditional Correlation Multivariate Garch. NBER, Working Paper No. w8554.

Engle R.F. (2002). Dynamic Conditional Correlation: A simple class of multivariate generalized autoregressive conditional heteroskedasticity models. Journal of Business and Economic Statistics, $20(3), 339-350$.

Engle, R.F., Ito, T., \& Lin, W.L. (1990). Meteor Showers or Heat Waves? Heteroskedastic Intra-daily Volatility in the Foreign Exchange Market. Econometrica, 58(3), 525-42. 
Engle, R.F., \& Ng, V.K. (1993). Measuring and testing the impact of news on volatility. Journal of Finance, 48, 1749-1778.

Engle, R.F., \& Susmel, R. (1994). Hourly volatility spillovers between international equity markets. Journal of International Money and Finance, 13(1), 23-25.

Fraser, P., \& Power, D. (1997). Stock return volatility and information: An empirical analysis of Pacific Rim, UK and US equity markets. Applied Financial Economics, 7(3), 241-253.

Fratzscher, M. (2002). Financial Market Integration in Europe: On effects of EMU on stock markets. International Journal of Finance and Economics, 7(3), 165-193.

Gallant, A.R., Rossi, P.E., \& Tauchen, G. (1993). Nonlinear dynamic structures. Econometrica, 61, 871-907

Glosten, L.R., Jagannathan, R., \& Runkle, D.E. (1993). On the relation between the expected value and the volatility of the nominal excess return of stocks. The Journal of Finance, 48(5), 1779-1801.

Guzmán, D.S., Ramírez, F.H., \& Romero, Y.P. (2013). Mercado Integrado Latinoamericano (MILA): análisis de correlación y diversificación de los portafolios de acciones de los tres países miembros en el periodo 2007-2012. [Trabajo de Grado de la maestría en Finanzas]. Universidad de Medellín, Bogotá.

Hamao, Y., Masulis, R.W., \& Ng, V. (1990). Correlations in price changes and volatility across international stock markets. Review of Financial Studies, 3(2), 281-307.

Hamao, Y., Masulis, R.W., Ng, V. (1991). The effect of the 1987 stock crash on international financial integration. In: Ziemba, W. Bailey, W. Hamao, Y. (Eds.), Japanese Financial Market Research. Elsevier, Amsterdam, 483-502.

Hamori, S., \& Imamura, Y. (2000). International transmission of stock prices among G7 countries: LAVAR approach. Applied Economics Letters, 7(9), 613-618.

Heston, S.L., Rouwenhorst, K., \& Wessels, R. E. (1995). The structure of international stock returns and the integration of capital markets. Journal of Empirical Finance, 2(3), 173-197.

Heytens, P.J. (1986). Testing market integration. Food Research Institute Studies, 2(1387-2016115927), 25-41.

Histórica caída de la Bolsa de Valores de Colombia. (2006). [En línea] Caracol. Recuperado de https://caracol.com.co/radio/2006/06/08/economia/1149778200\296067.html.

Kanas, A. (1998a). Volatility spillovers across equity markets: European evidence. Applied Financial Economics, 8(3), 245-256.

Kanas, A. (1998b). Linkages between the US and European equity markets: Further evidence from cointegration tests. Applied Financial Economics, 8(6), 607-14.

Kansa, K. (1992). Common stochastic trends in international stock markets. Journal of Monetary Economics, 29(1), 95-124.

King, M., Sentana, E., \& Wadhwani, S. (1994). Volatility and Links between National Stock Markets. Econometrica, 62(4), 901-33. 
Koop, G., Pesaran, M.H., \& Porter, S. M. (1996). Impulse Response Analysis in Nonlinear Multivariate Models. Journal of Economatrics, 74(1), 119-147.

Lahrech, A., \& Sylwester, K. (2011). U.S. and Latin American stock market linkages. Journal of International Money and Finance, 30, 1341-1357.

Najeeb, S.F., Bacha, O., \& Masih, M. (2015). Does heterogeneity in investment horizons affect portfolio diversification? Some insights using M-GARCH-DCC and wavelet correlation analysis. Emerging Markets Finance and Trade, 51(1), 188-208.

Pesaran, H.H., \& Shin, Y. (1998). Generalized Impulse Response Analysis in Linear Multivariate Models. Economics Letters, 58(1), 17-29.

Phillips, P.C., \& Perron, P. (1988). Testing for a unit root in time series regression. Biometrika, 75, 335-346.

Piffaut, P.V., \& Miró, D.R. (2016). Integración, contagio financiero y riesgo bursátil: ¿qué nos dice la evidencia empírica para el periodo 1995-2016. Cuadernos de Economía, 39(111), 138-147.

Richards, A.J. (1995). Comovements in international stock markets returns: Evidence of predictability but no cointegration. Journal of Monetary Economics, 36(3), 631-654.

Schmiedel, H., \& Schonenberger, A. (2005). Integration of Securities Market Infraestructures in the Euro Area. Ocasional Paper Series, 33.

Sim, C. (1980). Macroeconomics and reality. Econometrica, 48, 1-48.

Syllignakis, M.N., \& Kouretas, G.P. (2010). German, US and Central and Eastern European stock market integration. Open Economies Review, 21(4), 607-628.

Syllignakis, M. N., \& Kouretas, G.P. (2011). Dynamic correlation analysis of financial contagion: Evidence from the Central and Eastern European markets. International Review of Economics and Finance, 20(4), 717-732.

Tamanes, R. (1990). Estructura económica internacional. Madrid, España: Alianza Editorial.

Zakoian, J. M. (1994). Threshold heteroskedasticity models. Journal of Economic Dynamics and Control, 18, 931-955. 


\section{Anexo.}

a. Resultados modelo ARMA y GARCH univariados COLCAP.

Tabla 8. Resultados según los criterios de información de los modelos considerados para los rendimientos del COLCAP.

\begin{tabular}{|l|l|l|}
\hline \multicolumn{3}{|c|}{ Criterio de información: COLCAP } \\
\hline Modelo & AIC & BIC \\
\hline AR(2) & -3568.171 & -3554.021 \\
\hline MA(2) & -3568.082 & -3553.932 \\
\hline ARMA(1,2) & -3566.967 & -3543.384 \\
\hline ARMA(2,1) & -3566.739 & -3543.156 \\
\hline
\end{tabular}

Fuente: Elaboración propia.

Tabla 9. Resultados de las estimaciones del modelo AR (2) para los rendimientos del COLCAP que incluye los coeficientes estimados y los errores estándar.

\begin{tabular}{|l|l|l|l|}
\hline \multicolumn{4}{|c|}{ Estimación Modelo AR(2) } \\
\hline & Coeficiente & Desv.Estándar & P-valor \\
\hline$\mu$ & 0.0028847 & 0.0011942 & 0.016 \\
\hline$\phi_{2}$ & 0.1300093 & 0.0225781 & 0.000 \\
\hline$\sigma$ & 0.027806 & 0.000297 & 0.000 \\
\hline
\end{tabular}

Este modelo fue escogido de acuerdo con los criterios de información. Fuente: Elaboración propia.

Tabla 10. Correlograma de los residuales. Se muestra el rezago y el p-value del estadístico $Q$ de la prueba de Ljung-Box (1978) de autocorrelación serial.

\begin{tabular}{|c|c|}
\hline \multicolumn{2}{|c|}{ Correlograma de los residuales } \\
\hline Rezago & P-valor \\
\hline 1 & 0.7606 \\
\hline 2 & 0.9546 \\
\hline 3 & 0.3871 \\
\hline 4 & 0.5494 \\
\hline 5 & 0.6917 \\
\hline 10 & 0.5716 \\
\hline 20 & 0.4439 \\
\hline 30 & 0.5695 \\
\hline 40 & 0.0779 \\
\hline
\end{tabular}

No se rechaza la hipótesis nula de ausencia de correlación de cualquier orden. Fuente: Elaboración propia.

Tabla 11. Resultados según los criterios de información de los modelos considerados de la familia ARCH para los rendimientos del COLCAP.

\begin{tabular}{|l|l|l|}
\hline \multicolumn{3}{|c|}{ Criterio de información: COLCAP } \\
\hline Modelo & AIC & BIC \\
\hline ARCH(1) & -3618.418 & -3599.552 \\
\hline GARCH(1,1) & -3707.265 & -3683.682 \\
\hline GJR-ARCH(1,1) & -3629.798 & -3606.215 \\
\hline GJR-GARCH(1,1) & -3708.445 & -3680.145 \\
\hline
\end{tabular}

Fuente: Elaboración propia. 
Tabla 12. Resultados del modelo ARMA-GARCH univariado definitivo para los rendimientos del COLCAP.

\begin{tabular}{|c|c|c|c|}
\hline \multicolumn{4}{|c|}{ Estimación modelo AR(2) ARCH(1) GJR(1) GARCH(1) } \\
\hline & Coeficiente & Desv.Estándar & P-valor \\
\hline$\mu$ & 0.0029176 & 0.0010236 & 0.004 \\
\hline$\phi_{2}$ & 0.1082556 & 0.0378587 & 0.004 \\
\hline$\alpha$ & 0.3118807 & 0.0378229 & 0.000 \\
\hline$\gamma$ & -0.1147931 & 0.0427219 & 0.007 \\
\hline$\beta$ & 0.6448025 & 0.0465835 & 0.000 \\
\hline$\omega$ & 0.0001 & 0.0000231 & 0.000 \\
\hline
\end{tabular}

Fuente: Elaboración propia.

Tabla 13. Se muestra el rezago y el p-value del estadístico $Q$ de la prueba de Ljung-Box (1978) de autocorrelación serial.

\begin{tabular}{|r|l|}
\hline \multicolumn{2}{|c|}{ Correlograma de los residuales } \\
\hline Rezago & P-valor \\
\hline 1 & 0.7876 \\
\hline 2 & 0.7896 \\
\hline 3 & 0.3368 \\
\hline 4 & 0.4963 \\
\hline 5 & 0.6408 \\
\hline 10 & 0.5643 \\
\hline 20 & 0.4689 \\
\hline 30 & 0.5930 \\
\hline 40 & 0.0726 \\
\hline
\end{tabular}

No se rechaza la hipótesis nula de ausencia de correlación de cualquier orden.

Fuente: Elaboración propia.

b. Resultados modelo ARMA y GARCH univariados IPSA.

Tabla 14. Resultados según los criterios de información de los modelos para los rendimientos del IPSA.

\begin{tabular}{|c|c|c|}
\hline \multicolumn{3}{|c|}{ Criterio de información: IPSA } \\
\hline Modelo & AIC & BIC \\
\hline AR(1) & -3834.678 & -3820.529 \\
\hline MA(1) & -3834.63 & -3820.48 \\
\hline ARMA(2,2) & -3840.396 & -3812.097 \\
\hline
\end{tabular}

Fuente: Elaboración propia.

Tabla 15. Resultados de las estimaciones del modelo AR (1) para los rendimientos del IPSA.

\begin{tabular}{|c|c|c|c|}
\hline \multicolumn{4}{|c|}{ Estimación Modelo AR(1) } \\
\hline & Coeficiente & Desv.Estándar & P-valor \\
\hline$\mu$ & 0.0021449 & 0.0008839 & 0.015 \\
\hline$\phi_{1}$ & -0.0379283 & 0.0159358 & 0.017 \\
\hline$\sigma$ & 0.0236643 & 0.000239 & 0.000 \\
\hline
\end{tabular}

Este modelo fue el escogido por los criterios de información.

Fuente: Elaboración propia. 
Tabla 16. Correlograma de los residuales. Se muestra el rezago y el p-valor del estadístico $Q$ de la prueba de Ljung-Box (1978) de autocorrelación serial.

\begin{tabular}{|c|c|}
\hline \multicolumn{2}{|c|}{ Correlograma de los residuales } \\
\hline Rezago & P-valor \\
\hline 1 & 0.9842 \\
\hline 2 & 0.8356 \\
\hline 3 & 0.8756 \\
\hline 4 & 0.1592 \\
\hline 5 & 0.2477 \\
\hline 10 & 0.1925 \\
\hline 20 & 0.2591 \\
\hline 30 & 0.1501 \\
\hline 40 & 0.1712 \\
\hline
\end{tabular}

No se rechaza la hipótesis nula de ausencia de correlación de cualquier orden.

Fuente: Elaboración propia.

Tabla 17. Resultados según criterios de información de los modelos considerados de la familia ARCH para los rendimientos de IPSA.

\begin{tabular}{|l|l|l|}
\hline \multicolumn{3}{|c|}{ Criterio de información: IPSA } \\
\hline Modelo & AIC & BIC \\
\hline ARCH(1) & -3955.365 & -3941.215 \\
\hline GARCH(1,1) & -4003.049 & -3984.183 \\
\hline GJR-ARCH(1,1) & -3969.106 & -3950.24 \\
\hline GJR-GARCH(1,1) & -4019.81 & -3996.227 \\
\hline \multicolumn{2}{|c|}{ Fuente: Elaboración propia } \\
\hline
\end{tabular}

Fuente: Elaboración propia.

Tabla 18. Resultados del modelo ARMA-GARCH univariado definitivo para los rendimientos del IPSA.

\begin{tabular}{|c|c|c|c|}
\hline \multicolumn{4}{|c|}{ Estimación modelo ARCH(1) GJR(1) GARCH(1) } \\
\hline & Coeficiente & Desv.Estándar & P-valor \\
\hline$\mu$ & 0.0021237 & 0.0007267 & 0.003 \\
\hline$\alpha$ & 0.2404778 & 0.0259998 & 0.000 \\
\hline$\gamma$ & -0.1891288 & 0.0312415 & 0.000 \\
\hline$\beta$ & 0.7550667 & 0.0269506 & 0.000 \\
\hline$\omega$ & 0.0000504 & 0.000009 & 0.000 \\
\hline
\end{tabular}

Fuente: Elaboración propia.

Tabla 19. Correlograma de los residuales. Se muestra el rezago y el p-value del estadístico $Q$ de la prueba de Ljung-Box (1978) de autocorrelación serial.

\begin{tabular}{|c|c|}
\hline Correlograma de los residuales \\
\hline Rezago & P-valor \\
\hline 1 & 0.2740 \\
\hline 2 & 0.4552 \\
\hline 3 & 0.5751 \\
\hline 4 & 0.0939 \\
\hline 5 & 0.1578 \\
\hline 10 & 0.1430 \\
\hline 20 & 0.1890 \\
\hline 30 & 0.1071 \\
\hline 40 & 0.1402 \\
\hline
\end{tabular}

No se rechaza la hipótesis nula de ausencia de correlación de cualquier orden.

Fuente: Elaboración propia. 
c. Resultados modelo ARMA y GARCH univariados IPC.

Tabla 20. Resultados según los criterios de información de los modelos considerados para los rendimientos del IPC.

\begin{tabular}{|l|l|l|}
\hline \multicolumn{3}{|c|}{ Criterio de información: IPC } \\
\hline Modelo & AIC & BIC \\
\hline ARMA(1,1) & -3622.934 & -3604.068 \\
\hline AR(2) & -3623.048 & -3604.182 \\
\hline MA(2) & -3622.626 & -3603.76 \\
\hline
\end{tabular}

Fuente: Elaboración propia.

Tabla 21. Resultados de las estimaciones del modelo AR (2) para los rendimientos del IPC.

\begin{tabular}{|c|c|c|c|}
\hline \multicolumn{4}{|c|}{ Estimación Modelo AR(2) } \\
\hline & Coeficiente & Desv.Estándar & P-valor \\
\hline$\mu$ & 0.00239 & 0.0009966 & 0.016 \\
\hline$\phi_{1}$ & -0.109507 & 0.019897 & 0.000 \\
\hline$\phi_{2}$ & 0.0601237 & 0.0235789 & 0.011 \\
\hline$\sigma$ & 0.0268644 & 0.0268644 & 0.000 \\
\hline
\end{tabular}

Este modelo fue escogido según los criterios de información.

Fuente: Elaboración propia.

Tabla 22. Correlograma de los residuales.

\begin{tabular}{|c|c|}
\hline \multicolumn{2}{|c|}{ Correlograma de los residuales } \\
\hline Rezago & P-valor \\
\hline 1 & 0.7606 \\
\hline 2 & 0.9546 \\
\hline 3 & 0.3871 \\
\hline 4 & 0.5494 \\
\hline 5 & 0.6917 \\
\hline 10 & 0.5716 \\
\hline 20 & 0.4439 \\
\hline 30 & 0.5695 \\
\hline 40 & 0.0779 \\
\hline
\end{tabular}

Se muestra el rezago y el p-value del estadístico Q de la prueba de Ljung-Box (1978) de autocorrelación serial. No se rechaza la hipótesis nula de ausencia de correlación de cualquier orden.

Fuente: Elaboración propia.

Tabla 23. Resultados de los criterios de información de los modelos considerados de la familia ARCH para los rendimientos de IPC.

\begin{tabular}{|l|l|l|}
\hline \multicolumn{3}{|c|}{ Criterio de información: IPC } \\
\hline Modelo & AIC & BIC \\
\hline ARCH(1) & -3706.868 & -3688.001 \\
\hline GARCH(1,1) & -3823.022 & -3799.439 \\
\hline GJR-GARCH(1,1) & -3833.18 & -3804.88 \\
\hline \multicolumn{3}{|c|}{ Fuente: Elaboración propia. }
\end{tabular}


Tabla 24. Resultados del modelo ARMA-GARCH univariado definitivo para los rendimientos del IPC.

\begin{tabular}{|c|c|c|c|}
\hline \multicolumn{4}{|c|}{ Estimación modelo AR(1) ARCH(1) GJR(1) GARCH(1) } \\
\hline & Coeficiente & Desv.Estándar & P-valor \\
\hline$\mu$ & 0.0020242 & 0.0007705 & 0.009 \\
\hline$\phi_{1}$ & -0.0674781 & 0.0386916 & 0.081 \\
\hline$\alpha$ & 0.1535968 & 0.0258197 & 0.000 \\
\hline$\gamma$ & -0.1122873 & 0.0258089 & 0.000 \\
\hline$\beta$ & 0.8499575 & 0.0269506 & 0.000 \\
\hline$\omega$ & 0.000031 & 0.000008 & 0.001 \\
\hline
\end{tabular}

Fuente: Elaboración propia.

Tabla 25. Correlograma de los residuales. Se muestra el rezago y el p-value del estadístico $Q$ de la prueba de Ljung-Box (1978) de autocorrelación serial.

\begin{tabular}{|r|l|}
\hline \multicolumn{2}{|c|}{ Correlograma de los residuales } \\
\hline Rezago & P-valor \\
\hline 1 & 0.1980 \\
\hline 2 & 0.0804 \\
\hline 3 & 0.1263 \\
\hline 4 & 0.1969 \\
\hline 5 & 0.2914 \\
\hline 10 & 0.2430 \\
\hline 20 & 0.0385 \\
\hline 30 & 0.1137 \\
\hline 40 & 0.1137 \\
\hline
\end{tabular}

No se rechaza la hipótesis nula de ausencia de correlación de cualquier orden.

Fuente: Elaboración propia.

d. Resultados modelo ARMA y GARCH univariados IGBVL.

Tabla 26. Resultados de las estimaciones del modelo ARMA $(4,4)$ para los rendimientos del IGBVL.

\begin{tabular}{|c|c|c|c|}
\hline \multicolumn{5}{|c|}{ Estimación modelo ARMA(4,4) } \\
\hline & Coeficiente & Desv.Estándar & P-valor \\
\hline$\mu$ & 0.0034859 & 0.0024291 & 0.151 \\
\hline$\phi_{1}$ & 0.0327887 & 0.2782819 & 0.906 \\
\hline$\phi_{2}$ & 0.1509578 & 0.0742789 & 0.042 \\
\hline$\phi_{3}$ & 0.8486822 & 0.0366776 & 0.000 \\
\hline$\phi_{4}$ & -0.1802145 & 0.2286626 & 0.431 \\
\hline$\theta_{1}$ & 0.0826424 & 0.2799629 & 0.768 \\
\hline$\theta_{2}$ & -0.0303985 & 0.0968 & 0.753 \\
\hline$\theta_{3}$ & -0.8258729 & 0.0664987 & 0.000 \\
\hline$\theta_{4}$ & 0.0522762 & 0.2066667 & 0.800 \\
\hline$\sigma$ & 0.0339599 & 0.0004512 & 0.000 \\
\hline
\end{tabular}

Este modelo fue escogido según los criterios de información.

Fuente: Elaboración propia.

Tabla 27. Correlograma de los residuales. Se muestra el rezago y el p-valor del estadístico Q de la prueba de Ljung-Box(1978) de autocorrelación serial.

\begin{tabular}{|c|c|}
\hline \multicolumn{2}{|c|}{ Correlograma de los residuales } \\
\hline Rezago & P-valor \\
\hline 1 & 0.9903 \\
\hline 2 & 0.9319 \\
\hline
\end{tabular}




\begin{tabular}{|c|c|}
\hline 3 & 0.5569 \\
\hline 4 & 0.6886 \\
\hline 5 & 0.5773 \\
\hline 10 & 0.1314 \\
\hline 20 & 0.4643 \\
\hline 30 & 0.3952 \\
\hline 40 & 0.2627 \\
\hline
\end{tabular}

No se rechaza la hipótesis nula de ausencia de correlación de cualquier orden.

Fuente: Elaboración propia.

Tabla 28. Resultados de los criterios de información de los modelos considerados de la familia ARCH para los rendimientos de IGBVL.

\begin{tabular}{|l|l|l|}
\hline \multicolumn{3}{|c|}{ Criterio de información: IGBVL } \\
\hline Modelo & AIC & BIC \\
\hline ARCH(1) & -3429.92 & -3378.037 \\
\hline GJR-GARCH(1,1) & -3475.432 & -3414.116 \\
\hline
\end{tabular}

Fuente: Elaboración propia.

Tabla 29. Resultados de las estimaciones del modelo ARMA-GARCH para los rendimientos del IGBVL.

\begin{tabular}{|c|c|c|c|}
\hline \multicolumn{4}{|c|}{ Estimación modelo AR(4) MA(3) ARCH(1) GARCH(1) } \\
\hline & Coeficiente & Desv.Estándar & P-valor \\
\hline$\mu$ & 0.003925 & 0.0018523 & 0.034 \\
\hline$\phi_{1}$ & 0.1359873 & 0.0420877 & 0.001 \\
\hline$\phi_{2}$ & 0.0869408 & 0.0246256 & 0.000 \\
\hline$\phi_{3}$ & 0.8408132 & 0.0540273 & 0.000 \\
\hline$\phi_{4}$ & -0.1519917 & 0.0365259 & 0.000 \\
\hline$\theta_{3}$ & -0.813883 & 0.0567216 & 0.000 \\
\hline$\alpha$ & 0.2995715 & 0.0320722 & 0.000 \\
\hline$\beta$ & 0.6436605 & 0.0335239 & 0.000 \\
\hline$\omega$ & 0.000098 & 0.0000177 & 0.000 \\
\hline
\end{tabular}

Fuente: Elaboración propia.

Tabla 30. Se muestra el rezago y el p-valor del estadístico $Q$ de la prueba de Ljung-Box (1978) de autocorrelación serial.

\begin{tabular}{|c|c|}
\hline \multicolumn{2}{|c|}{ Correlograma de los residuales } \\
\hline Rezago & P-valor \\
\hline 1 & 0.4379 \\
\hline 2 & 0.4715 \\
\hline 3 & 0.3327 \\
\hline 4 & 0.4311 \\
\hline 5 & 0.5028 \\
\hline 10 & 0.0972 \\
\hline 20 & 0.1837 \\
\hline 30 & 0.2798 \\
\hline 40 & 0.1022 \\
\hline
\end{tabular}

No se rechaza la hipótesis nula de ausencia de correlación de cualquier orden.

Fuente: Elaboración propia. 